\title{
PROBLEMS IN FOLIATIONS AND LAMINATIONS OF 3-MANIFOLDS
}

\author{
DANNY CALEGARI
}

\section{INTRODUCTION AND BASIC DEFINITIONS}

1.1. Notation. I will try to use consistent notation throughout, so for instance, $\mathscr{F}, \mathscr{G}$ denote foliations, $\Lambda$ denotes a lamination, $\lambda, \mu, \nu$ denote leaves, etc. For a given object $X$ in a manifold $M, \widetilde{X}$ will denote the pullback of $X$ to the universal cover $\widetilde{M}$.

1.2. Attribution. I have tried to credit questions to their originators. There are certain caveats to this, however:

- I have made no attempt to verify that my sources were themselves original.

- I have occasionally edited or reformulated questions; in so doing, it is possible that I have perverted the intent of the originator.

- I have not systematically checked other problem lists to see if the questions here were posed independently elsewhere.

1.3. Other problem lists. The format and taste of this problem list is greatly influenced by Gabai's well known problem list [45]. However I have tried to choose questions which complement, rather than overlap with, Gabai's choice of problems.

1.4. Basic objects. A reference for the basic theory of foliations is [24]. An overview of the state of the subject as it stood in 1990 is contained in [42]. [45] also contains background and numerous examples.

Definition 1.1. A codimension one foliation $\mathscr{F}$ of a 3 -manifold $M$ is taut if there is a circle $\gamma$ transverse to $\mathscr{F}$ intersecting every leaf.

A basic reference is [41]. Note that a foliation of an atoroidal 3-manifold is taut iff it contains no torus leaf.

For a taut foliation $\mathscr{F}$ of $M, \widetilde{M}$ may be taken to be an open subset of $\mathbb{R}^{3}$ so that $\widetilde{F}$ consists of the intersection of $\widetilde{M}$ with the horizontal planes in $\mathbb{R}^{3}$. These leaves are all disks, and the leaf space $L$ of $\widetilde{\mathscr{F}}$ is a simply-connected 1 -manifold. There is a canonical holonomy representation

$$
\rho_{H}: \pi_{1}(M) \rightarrow \operatorname{Homeo}(L)
$$

Definition 1.2. A taut foliation is said to be or have

- $\mathbb{R}$-covered if the leaf space of $\widetilde{\mathscr{F}}$ is homeomorphic to $\mathbb{R}$.

- one-sided branching if the leaf space of $\widetilde{F}$ branches in one direction.

- two-sided branching if the leaf space of $\widetilde{F}$ branches in both directions.

All possibilities are realized, for foliations of atoroidal 3-manifolds.

Date: $9 / 8 / 2002$. Version 0.78 . 
Definition 1.3. A codimension one lamination $\Lambda$ in a 3-manifold $M$ is a foliation by twodimensional leaves of a closed subset of $M$. A lamination is essential if it contains no sphere leaf or torus leaf bounding a solid torus, if complementary regions are irreducible and have incompressible boundary, and admit no compressing monogons.

A taut foliation is an example of an essential lamination. A closed union of leaves of a taut foliation is another example.

Definition 1.4. The complementary regions to an essential lamination fall into two kinds of pieces, which can be chosen uniquely up to isotopy: $I$-bundles $\Sigma_{i} \times I$ over noncompact surfaces $\Sigma_{i}$, called interstitial regions, and compact pieces called guts $\mathfrak{G}_{i}$ meeting the $I-$ bundles along annuli called interstitial annuli. An essential lamination is genuine if it has nonempty gut - i.e. if some complementary region is not an $I$-bundle.

To construct the partition of a complementary region $C$ into guts and interstices, take as a first approximation of the interstices the characteristic $I$-bundle of $C, \partial C$, and then remove all $I$-bundles over non-compact components.

For more details on essential and genuine laminations, see [52], [48] and [50]. Note that though foliations can always be co-oriented in some finite cover of the ambient manifold, laminations admit local obstructions to co-orientability.

Definition 1.5. A genuine lamination has solid torus guts if all gut regions are neutered ideal polygon bundles over $S^{1}$. It is full if some complementary region is an ideal polygon bundle over $S^{1}$. It is very full if every complementary region is a bundle region.

There is a procedure to turn co-oriented very full genuine laminations into taut foliations, by filling in the complementary regions with monkey saddles. These are disks which are asymptotic along the boundary leaves of a complementary region in alternating directions. The complementary regions can be given a bundle structure over $S^{1}$ where these saddle leaves are the fibers.

Definition 1.6. A pseudo-Anosov flow $X$ on a manifold $M$ is a flow which, away from a finite number of closed orbits $\gamma_{i}$, is Anosov - that is, there is a decomposition of $T M$ as a sum $E_{s} \oplus E_{u} \oplus T X$ where the time $t$ flow contracts $E_{u}$ by some factor $\lambda^{-t}$ and expands $E_{s}$ by $\lambda^{t}$, for some $\lambda>0$. Along the singular orbits, the local picture is that of a semi-branched cover of an Anosov orbit of degree $n / 2$ for $n \geq 3$. The distributions $E_{s}$ and $E_{u}$ are integrable, and integrate to give two singular foliations $\mathscr{F}_{s}, \mathscr{F}_{u}$ the stable and unstable integral foliations. If one splits open $\mathscr{F}_{s}, \mathscr{F}_{u}$ along the singular leaves and takes the closure, one obtains very full genuine laminations $\Lambda_{s}, \Lambda_{u}$.

The basic examples of pseudo-Anosov flows are the suspensions of pseudo-Anosov automorphisms of surfaces. See [116].

Definition 1.7. A lamination $\Lambda$ has hyperbolic leaves if the leaves of $\widetilde{\Lambda}$ are (uniformly) quasi-isometric to $\mathbb{H}^{2}$ with the path metric induced from some metric on $M$.

A theorem of Candel (23]) implies that for $M$ atoroidal, any essential lamination $\Lambda$ can be given a metric of constant curvature -1 . In particular, all such laminations have hyperbolic leaves.

Definition 1.8. Suppose $\mathscr{F}$ is a taut foliation of $M$ with hyperbolic leaves. A universal circle for $\mathscr{F}$ is a circle $S_{\text {univ }}^{1}$ with a group action

$$
\rho_{\text {univ }}: \pi_{1}(M) \rightarrow \operatorname{Homeo}\left(S_{\text {univ }}^{1}\right)
$$

satisfying the following properties: 
(1) For all leaves $\lambda$ of $\widetilde{\mathscr{F}}$ there is a monotone map

$$
\phi_{\lambda}: S_{\text {univ }}^{1} \rightarrow S_{\infty}^{1}(\lambda)
$$

where $\phi_{\lambda}$ varies continuously as a function of $\lambda$. Here a map between circles is monotone if the preimage of every point is contractible.

(2) For each leaf $\lambda$ of $\widetilde{\mathscr{F}}$ and each $\alpha \in \pi_{1}(M)$ the following diagram commutes:

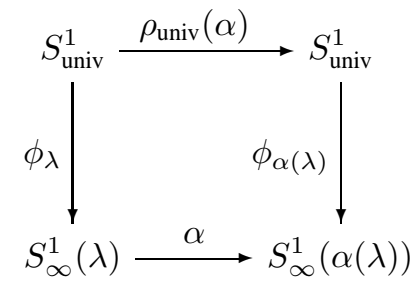

(3) For any leaf $\lambda$, the associated gaps are the maximal connected open intervals in $S_{\text {univ }}^{1}$ mapped to points by $\phi_{\lambda}$. The complement of the gaps in $S_{\text {univ }}^{1}$ is the core associated to $\lambda$. Then for any pair $\mu, \lambda$ of incomparable leaves, the core associated to $\lambda$ is contained in a single gap associated to $\mu$, and vice versa.

Universal circles are introduced in [118]; examples and discussion are found in [15], [18] and [14. Since the map between the universal circle and $S_{\infty}^{1}(\lambda)$ is monotone for each $\lambda$, it easily follows that the Euler class of the representation $\rho_{\text {univ }}$ is equal to the Euler class of $T \mathscr{F}$.

Definition 1.9. A branched surface $\mathscr{B}$ is a certain kind of 2 -complex in a 3 -manifold with a well-defined $C^{1}$ tangent space everywhere. The place where the branched surface is not a surface is called the branch locus; it is a 1-complex, and the branch locus is not a 1-manifold only at isolated points, where two lines of the branch locus cross each other transversely.

Along the nonsingular part of the branch locus of a branched surface, there is a natural transverse orientation, which points in the direction in which the two branches come together. Where two arcs of the branch locus cross, there is one complementary quadrant where both co-orientations are inward, and one where both co-orientations are outward.

Definition 1.10. A sink disk for a branched surface is an embedded disk $D \subset \mathscr{B}$ with boundary contained in the branch locus, such that the transverse orientation points inward all along $\partial D$.

Sink disks are introduced by Li in [80]. He shows, amongst other things, that branched surfaces without sink disks fully carry essential laminations, and that every essential lamination (with some very simple exceptions) is carried by a branched surface without sink disks.

1.5. 3-manifold topology. Throughout this paper, numerous reference is made, implicitly or explicitly, to elements of the theory of 3-manifolds. A basic reference for this theory is [70]. A reference for the theory of geometrization is [114].

1.6. Acknowledgements. Many thanks to Charles Delman, Tao Li, Curt McMullen, Saul Schleimer and participants in the problem session on foliations and laminations at the 2001 Georgia International Topology Conference. Ian Agol and Nathan Dunfield should be singled out for special thanks, for their considerable contributions to this problem list. 


\section{EXISTENCE QUESTIONS}

Question 2.1. Which hyperbolic 3-manifolds admit taut foliations? Give an effective procedure to decide if a hyperbolic 3-manifold admits a taut foliation. For a useful property $\mathrm{p}$ of a 3-manifold, give an explicit construction of infinitely many 3-manifold with property $\mathrm{p}$ with/without a taut foliation. Same question for essential laminations.

Remark. (1) It is known by [103] that there exist infinitely many hyperbolic 3-manifolds without taut foliations. These manifolds are so special that their fundamental groups admit no nontrivial actions on (potentially non-Hausdorff) 1-manifolds. Here an action on a non-Hausdorff 1-manifold is trivial if it has a global fixed point on the maximal Hausdorff quotient. The proof is somewhat ad hoc and relies on the special structure of the fundamental groups of these manifolds (they are Dehn fillings of certain punctured torus bundles with negative trace). It should be remarked that Hatcher initially speculated that such manifolds would be good candidates to be taut foliation free.

The method of proof is not entirely satisfactory for two reasons. Firstly, the certificate that the manifolds in question admit no taut foliation is very long, and it seems unlikely that it could be further compressed within a similar approach. (See [22] for a different method which provides a much shorter certificate that the Weeks manifold admits no taut foliation). Secondly, the criterion by which the existence of taut foliations is ruled out is not a priori sharp. For instance, if $f: M \rightarrow N$ has positive degree and $N$ admits a taut foliation $\mathscr{F}$, then $\pi_{1}(M)$ acts on the leaf space of $\widetilde{F}$ without a global fixed point; on the other hand, it is unclear whether such an $M$ admits a taut foliation in general. In particular, it is important to understand when a 3 -manifold admitting a nontrivial action on a non-Hausdorff 1-manifold admits a taut foliation.

The methods of [103] are extended by Fenley in [38] to show that infinitely many of these hyperbolic manifolds actually admit no essential laminations, by showing that their fundamental groups admit no nontrivial actions on order trees. It suffers from the same shortcomings as [103], and is therefore perhaps more of theoretical and philosophical than of practical interest.

(2) Work of Agol [2] gives strong restrictions on the topology of the guts of a tight genuine lamination on a hyperbolic manifold $M$ of small volume. If a taut foliation $\mathscr{F}$ is not monotone equivalent to a minimal foliation, some minimal set is a genuine lamination. Thus it might be interesting to give examples of 3-manifolds without taut foliations of large volume and/or injectivity radius.

(3) Agol and Li [3], combining work of Li [80] and Brittenham [10], give an algorithm to decide whether a given 3-manifold admits an essential lamination. For, if a 3-manifold admits a nowhere dense essential lamination, Brittenham shows it admits an essential lamination in normal form with respect to any triangulation, and therefore is carried by one of a finite constructible set of branched surfaces. With more work, using technology of Gabai [46], one can show that every essential nowhere dense lamination is fully carried by one of a finite constructible set of branched surfaces. Li shows that every essential lamination can, after blowing up, be carried by a branched surface without a sink disk (except for the special case of a foliation of $T^{3}$ by planes). So the algorithm proceeds by taking each of the finite constructed set of branched surfaces, and splits it open repeatedly in all possible ways. After some finite time, either every branched surface reveals an obstruction 
to being fully split open, or some branched surface can be split open to a branched surface with no sink disk. How computationally efficient is this algorithm? Is there any case in which it might actually be implemented on a computer, e.g. for the Seifert-Weber dodecahedral space?

Note that the method of Agol and Li decides whether a given 3-manifold admits a taut foliation. For, one can check that one of the finite constructible set of branched surfaces which fully carries an essential lamination has $I$-bundle complementary region.

(4) Properties $\mathrm{p}$ for which this question is interesting might include Hakenness, or more generally, laminar properites. Obstructions to the existence of a taut foliation which are homological in nature probably do not obstruct the existence of genuine laminations. In particular, the local non co-orientability is a strong advantage. Since taut foliations can be perturbed to symplectically semi-fillable contact structures by Eliashberg and Thurston [32], one might be able to use contact or symplectic 4-manifold techniques (e.g. gauge theory) to rule out the existence of taut foliations on certain manifolds which contain genuine laminations. Conversely it would be very interesting to give an example of a hyperbolic 3-manifold with a semi-fillable contact structure but no taut foliation.

(5) For a given manifold $M$ and a hyperbolic knot $K \subset M$, the set of surgeries on $K$ giving rise to a tautly foliated or laminar manifold, if not everything, is probably quite complicated in some cases. There are probably arithmetic criteria (e.g. some persistent lamination might be co-orientable or not after certain surgeries and therefore fillable or otherwise), inequalities (e.g. branched surfaces supporting foliations realizing certain slopes), bounded cohomology (e.g. Milnor-Wood), etc. There are existence results of Gabai-Mosher [93] which say that if $K$ is a hyperbolic knot, there is a degeneracy slope $\delta$ associated to $K$ such that the stable and unstable laminations coming from a pseudo-Anosov flow on $M-K$ stay essential for surgery on all slopes $\alpha$ with $\Delta(\delta, \alpha)>1$. Bootstrap techniques to determine degeneracy slopes are exploited in [22] to give a partial table of which small volume hyperbolic manifolds are laminar.

Question 2.2. Is there an effective algorithmic procedure to produce and recognize a hyperbolic knot of depth $n$ for any given $n$ ? What about $\geq n$ ?

Remark. (1) Satellite knots of arbitrary depth were produced by Cantwell and Conlon. A single explicit example of a hyperbolic knot of depth $\geq 2$ is constructed by Kobayashi in [74]. Brittenham has a class of examples which are conjecturally of depth $\geq 2$. But what about higher depth? See also question 10.3 .

(2) Algorithms exist to detect whether a knot is of depth 0 ([69]) or of depth 1 but they are not very useful in practice. Part of the problem is that finding Thurston norm minimizing surfaces is NP complete by [5]. On the other hand, perhaps there is a fast algorithm to check that a foliation is of minimal depth. A "good" algorithm producing a knot of depth $n$ would also produce a certificate for this fact. The paper 107] may be relevant here.

(3) Lackenby's technique of taut ideal triangulations [77] is useful in practice for finding and especially certifying minimal genus representatives for relative homology classes in open manifolds with torus boundary. 
Question 2.3. Given a collection $\mathscr{C}$ of topological or geometric types of surface, what 3 -manifolds admit a taut foliation $\mathscr{F}$ whose leaves are all homeomorphic or coarsely quasi-isometric to an element of the collection $\mathscr{C}$ ?

Remark. (1) Every topological surface is a leaf of a foliation (even a taut foliation) of some 3-manifold $M$, by a construction of Cantwell and Conlon [25].

(2) The Reeb stability theorem implies that a foliation of a 3-manifold with a spherical leaf is a bundle (possibly over an orbifold). Moreover, taut foliations of hyperbolic 3 -manifolds do not contain torus or Klein bottle leaves.

(3) By a theorem of Candel [23], if $\mathscr{F}$ is a taut foliation of an atoroidal 3-manifold $M$, there is a metric on $M$ with respect to which every leaf has constant curvature -1 . In particular, the universal covers of all the leaves are isometric to $\mathbb{H}^{2}$. Moreover, if there is a leaf (of $\mathscr{F}$, not $\widetilde{\mathscr{F}}$ ) with amenable growth, then its closure supports an invariant transverse measure, and therefore contains leaves of polynomial growth, by Plante's theorem. In particular, if $M$ is a rational homology sphere, every leaf of an orientable/co-orientable taut foliation $\mathscr{F}$ has uniformly exponential growth.

(4) In great generality this question is probably not so interesting, but in some special cases it is probably quite interesting. For instance, if $\mathscr{C}$ consists just of a plane, $M$ must be $T^{3}$ and the foliation must be monotone equivalent to one by planes of irrational slope. If $\mathscr{C}$ consists of just planes and cylinders and $\mathscr{F}$ is $\mathbb{R}$-covered, either $M$ is Solv, or there are cylinders whose nontrivial loops are homotopic to each other. If one adds the geometric constraint that the shortest loops in the cylinders are of bounded length, if the ambient manifold is atoroidal and therefore has word-hyperbolic fundamental group, these loops must be homotopic by an annulus of controlled diameter etc.

(5) The character of this problem would be very different if one asked for foliations with the property that almost all leaves were homeomorphic or coarsely quasiisometric to an element of $\mathscr{C}$. If $\mu$ is a harmonic measure on a foliated space, then any leaf $\lambda$ in the support of $\mu$ is quasi-homogeneous; that is, any local geometric feature of $\lambda$ on any scale recurs with definite density in any sufficiently large subset of $\lambda$. This is more or less a consequence of the fact that the support of $\mu$ is roughly the subset where leafwise random walks are recurrent. In this vein, there is a very satisfying theorem of Ghys that for $\Lambda$ any (abstract) compact Riemann surface lamination, and $\mu$ any harmonic measure, then $\mu$-almost all leaves are homeomorphic to one of the following six possibilities:

- The plane

- The Loch-Ness monster

- The cylinder

- Jacob's ladder

- $S^{2}-$ Cantor set

- $S^{2}$ - Cantor set with countably many handles accumulating to every point in the removed set.

Ghys' theorem is proved in [55].

Question 2.4. Let $X$ be a vector field on a 3-manifold. When is there a foliation $\mathscr{F}$ of $M$ transverse to $X$ ?

Remark. (1) If $M$ is Seifert-fibered and $X$ is a vector field tangent to the circle fibers, there are many restrictions on the existence of $\mathscr{F}$. The Milnor-Wood inequality says that the Euler class of a foliated circle bundle is at most as large in absolute 
value as the Euler characteristic of the base orbifold [88], [123]. This result can be phrased in terms of bounded cohomology, as saying that the norm of the Euler class is equal to 1 [60], [117]. Moreover, results of Brittenham and Thurston [9][111] imply that any taut foliation of a Seifert-fibered manifold is either transverse to the circle fibers or contains "vertical" leaves - leaves foliated by circles. Complete necessary and sufficient conditions for the existence of a foliation transverse to the foliation by circles of a Seifert fibered 3-manifold were established by Naimi [94] building on results of Jankins and Neumann [73].

(2) By analogy with the circle bundle case, one can ask whether there exists a slithering of $M$ over $S^{1}$ for which the slithering map $Z$ is realized by time 1 flow along $X$. Define an equivalence relation $\sim$ on $M$ which identifies pairs of points which are an integer distance apart on a flow line of $X$ (parameterized by the flow speed). Then one can think of $M / \sim$ as a circle bundle over the non-commutative space $M / X$, the leaf space of $X$. One would like to prove an analogue of the Milnor-Wood inequality for some appropriate fundamental classes for $M / \sim$ and $M / X$. See [29]. One can vary the question by allowing (nonconstant) reparameterizations of $X$.

(3) More generally, one can study a homology theory closely related to the Godbillon homology of $X$, whose $i$-chains are equivalence classes of singular maps of simplices to $M$, where two such maps $\sigma_{1}, \sigma_{2}: \Delta \rightarrow M$ are equivalent if there is a homotopy $S: \Delta \times I \rightarrow M$ where $S(\cdot, 0)=\sigma_{1}(\cdot), S(\cdot, 1)=\sigma_{2}(\cdot)$ and $S(p, I)$ is contained in a flowline of $X$. What is the cohomology of the bounded cochains on this complex?

\section{RIGIDITY AND MODULI}

Question 3.1. Let $M$ be atoroidal. Is there a natural refinement of the polyhedral structure of the unit ball of the Thurston norm to a polyhedron $\mathscr{P}_{\mathscr{F}}$ whose faces parameterize the set of taut of foliations of $M$ in the following sense:

(1) Each open face c of $\mathscr{P}_{\mathscr{F}}$ corresponds to a pseudo-Anosov flow $X_{c}$ or equivalence classes of pseudo-Anosov flows. The taut foliations "parameterized" by $\mathscr{P}_{\mathscr{F}}$ can be isotoped to be transverse (or almost transverse) to $X_{c}$, or some sublamination is monotone equivalent into the stable or unstable singular foliation of $X_{c}$.

(2) Every geometric limit of taut foliations $\mathscr{F}_{i}$ associated to a cell $c$ should be associated to some cell of the closure $\bar{c}$.

(3) There should be a natural polyhedral map from $\mathscr{P}_{\mathscr{F}}$ to (some polyhedral subdivision of) the unit ball of the Thurston norm.

Remark. (1) Basic references for the Thurston norm are [115] and [96]. For depth 0 (surface bundles), fibered homology classes are a union of top dimensional faces, by [115]. For depth 1, a refinement of the polyhedral structure is necessary, but is accomplished by Cantwell and Conlon in ([26]). Their procedure applies to depth 1 foliations of sutured manifolds, and therefore inductively, should give a polyhedral parameterization of all finite depth foliations on a fixed manifold, up to some appropriate equivalence relation.

(2) For a given fixed manifold $M$, Gabai has shown in 46 there is a triangulation $\tau$ such that every taut foliation of $M$ can be put into normal form with respect to $\tau$. There is a natural polyhedral structure $\mathscr{P}_{\tau}$ on the space of all normal surfaces carried by $\tau$. $\mathscr{P}_{\mathscr{F}}$ should be intermediate between the unit ball for the Thurston norm and $\mathscr{P}_{\tau}$. 
Note for certain classes of foliations ( $\mathbb{R}$-covered, one-sided branching, minimal foliations etc.) there are existence results for transverse pseudo-Anosov flows and, in many cases, stability results which say that these flows are unique up to isotopy for small deformations of the foliations ([15], [18], [20]) . It seems plausible that a deformation of normal foliations which preserves the normal disk types in each simplex should be mild enough to ensure stability of a transverse flow. Note that for finite depth foliations one has the theorem of Lackenby that a taut sutured manifold hierarchy can be placed in a position resembling normal form, relative to any triangulation ([76]).

(3) Kronheimer and Mrowka ([75]) found a characterization of the unit ball of the dual Thurston norm by means of differential geometry. Is there some way to detect the depth of a taut foliation or the codimension of the face it corresponds to by geometric properties of an approximating tight contact structure?

(4) By Gabai and Mosher, there is a pseudo-Anosov flow almost transverse to any finite depth foliation of a hyperbolic 3-manifold [93]. For depth $>1$, there are choices involved in the construction of the flow; any natural equivalence relation on pseudo-Anosov flows would have to take this ambiguity into account. Mosher in particular has developed a sophisticated picture of the relationship between pseudo-Anosov and, more generally, $p A$ flows on 3-manifolds and the Thurston norm, for instance in the papers [90], [91], 92].

(5) Thurston's theorem that euler classes of taut foliations are contained in the unit ball of the dual Thurston norm follows from the existence of a universal circle for a taut foliation, and from the Milnor-Wood inequality. One potential source of polyhedra $\mathscr{P}_{\mathscr{F}}$ might be unit balls of $L_{1}$ norms on certain natural quotients of the vector space $\Omega^{1}(M)$ of 1 -forms on $M$. Even an infinite dimensional polyhedron parameterizing taut foliations would be very interesting.

Question 3.2 (McMullen). Generalize the Teichmüller polynomial from the fibered faces of the Thurston norm ball to the other faces (of some possibly generalized polyhedron, perhaps the polyhedron sought in question 3.1).

Remark. (1) A reference for the Teichmüller polynomial associated to a fibered face of the Thurston norm ball is [86]. The fibered face itself is the Newton polygon of the polynomial associated to that face; amongst other information, the polynomial encodes the (topological) entropy of the (topologically unique) transverse pseudoAnosov flow associated to the face. If the singular stable and unstable foliations of the pseudo-Anosov flow are co-orientable, similar information is encoded in Fried's dynamical zeta function [39].

(2) The entropy of the pseudo-Anosov flow arising from a surface bundle over a circle is intimately related to the entropy of the automorphism of the fiber. Loosely speaking, the Teichmüller polynomial is a kind of "characteristic polynomial" for the action of the monodromy on the space of invariant transverse measures for an invariant train track. [83] gives a nice exposition of these ideas.

(3) For a depth $n$ foliation where $n>0$, one has invariant laminations for the endperiodic automorphisms of the fibers of greatest depth (see e.g. [33]), and one can study the entropy of these automorphisms. Global genuine laminations which can be collapsed to pseudo-Anosov flows are built up from these laminations at lowest depth inductively. A transverse pseudo-Anosov flow $X$ for a fibration or a transversely measured foliation $\mathscr{F}$ is like a Teichmüller geodesic for the family of hyperbolic metrics on the leaves of $\mathscr{F}$ (see [86]). The action of $\pi_{1}(M)$ 
on this Teichmüller geodesic is conjugate to the action on the transverse measures in the appropriate projective class for the singular foliations associated to $X$. For more general (not transversely measured) foliations with transverse pseudoAnosov flows, there might exist refined polynomials with values in $\mathbb{Z}[G L(n, \mathbb{R})]$ for some appropriate representation of $G L(n, \mathbb{R})$ in $\operatorname{Homeo}(\mathbb{R})$. For instance, there are many interesting slitherings of hyperbolic 3-manifolds with transverse $S \widetilde{L(2, \mathbb{R})}$ structure, associated to branched covers over unit tangent bundles of hypebolic orbifolds, where the branch loci are timelike with respect to the usual Lorentz structure on $\operatorname{PSL}(2, \mathbb{R})$. See [117] or questions 8.4 and 8.7 .

\section{Minimal SURFACES}

Question 4.1. Suppose $\mathscr{F}$ is a taut foliation of $M$. Characterize the space of metrics on $M$ for which $\mathscr{F}$ can be isotoped to consist of minimal surfaces.

Remark. (1) For general results on minimal surfaces relevant to this question, see [67], [99], [106].

(2) By Sullivan [110] a $C^{2}$ foliation of a 3-manifold can be made minimal with respect to some Riemannian metric on the ambient manifold iff it is taut. This is equivalent to the existence of a nonsingular divergence free normal vector field i.e. one which is perpendicular to $T \mathscr{F}$ everywhere. Given any transverse nonsingular vector field $X$ for which $\mathscr{L}_{X} \omega=0$ for some nonsingular 3 -form $\omega$, one can find a metric on $M$ for which $X$ is normal and $\omega$ is the volume form.

(3) A minimal surface in a hyperbolic manifold has intrinsic curvature $\leq-1$ everywhere. By Gauss-Bonnet, this puts an upper bound on the area of a compact minimal surface. Suppose $\mathscr{F}$ is a taut foliation in a hyperbolic 3-manifold enjoying some comparable bound on the leafwise intrinsic curvature. If $\mathscr{F}$ admits a nontrivial invariant transverse measure $\mu$ (not necessarily of full support), one obtains nontrivial constraints on the growth rate and topology of leaves in the support of $\mu$.

(4) Ben Andrews has constructed an area-minimizing flow on surfaces with principle curvatures $<1$ to a minimal surface with the same properties. Thurston has observed that elliptic PDE flows generally fail to preserve integrability of plane fields, since after a short time, such flows tend to make structures real analytic; but there are many $C^{\infty}$ foliations, even taut foliations, which admit no real analytic structure, and even manifolds which admit no (nontrivial) transversely real analytic foliation at all. Even so, it might be interesting to understand the behaviour of the leaves of $\mathscr{F}$ under Andrews' flow.

(5) A minimal surface in $\mathbb{E}^{3}$ or $\mathbb{H}^{3}$ has a natural real analytic structure, which one can think of as a kind of tangential rigidity condition. Zeghib shows in 125] that such tangential rigidity for foliations implies a transverse Lipschitz regularity; that is, any minimal foliation of $\mathbb{E}^{3}$ or $\mathbb{H}^{3}$ should be transversely Lipschitz. This already shows that certain taut foliations of hyperbolic 3-manifolds cannot be made minimal with respect to the hyperbolic metric, and perhaps can be used to show no foliation of a hyperbolic 3-manifold by minimal surfaces can occur. Notice in codimension 2 a minimal foliation would actually have to be geodesic; Zeghib showed that there are no nontrivial geodesic foliations of any codimension of any closed hyperbolic manifold ([124]). On the other hand, if $\mathscr{F}$ is proper and all leaves have the continuous extension property, one can inductively minimize 
leaves of fixed depth. The closure of the union will be an essential (but not genuine) lamination, monotone equivalent to the original foliation; but there seems to be no reason to expect it to be a foliation.

(6) One weakened version of the question is as follows: is there an a priori upper bound on the infimum of the mean curvature of a taut foliation $\mathscr{F}$ in a hyperbolic 3 -manifold $M$, independent of $\mathscr{F}$ and $M$ ?

Question 4.2. Given a collection of taut foliations $\mathscr{F}_{i}$ of $M$, what are the obstructions to finding a metric on $M$ for which the $\mathscr{F}_{i}$ (after an isotopy) are simultaneously minimal?

Remark. (1) Some kind of "stably minimal" property holds at the PL level. For foliations $\mathscr{F}_{i}$ whose tangent fields are sufficiently close as 2 -plane fields, we can find a triangulation $\tau$ of $M$ with respect to which the $\mathscr{F}_{i}$ can all be made simultaneously normal, in such a way that the co-orientations on the one skeleton of $\tau$ coming from the co-orientations on $\mathscr{F}_{i}$ agree. For sufficiently close $\mathscr{F}_{i}$, we can find a triangulation such that the 1-skeleton with this orientation is recurrent; such a 1skeleton admits a combinatorial weight $w: \tau^{1} \rightarrow \mathbb{Z}^{+}$such that for each vertex, the sum of the weights of the incoming edges is equal to the sum of the weights of the outgoing edges. With respect to such a weight, these foliations are "minimal" polyhedral surfaces. For similar ideas, see [67] and [47].

(2) Maybe this question become easier if we can replace the $\mathscr{F}_{i}$ by monotone equivalent laminations $\Lambda_{i}$. This is especially plausible if the $\mathscr{F}_{i}$ are finite depth - the leaves at each depth could be constructed inductively, and one could ignore the problem of filling up the remaining gaps with the leaves of greatest depth.

\section{REEB COMPONENTS}

Every closed 3-manifold admits a foliation with at most one Reeb component. One way to see this is to use an open-book decomposition. A more profound construction due to Gabai shows the core of the Reeb component can be essentially any knot (441]). Every open 3-manifold admits a foliation with possibly infinitely many Reeb components. This follows from Thurston's local construction of a foliation from an anti-orientation on a triangulation ([113]), and Moise's theorem that 3-manifolds can be triangulated.

Question 5.1. How many Reeb components must a foliation of an open 3-manifold contain?

Remark. (1) There are two questions here, a topological one and a geometric one. The topological question is perhaps not so interesting. To make the geometric question precise, some kind of quantification is necessary. First, the 3-manifold should have uniformly bounded geometry, and then one should uniformly bound the local geometry of the foliation, and either require all Reeb components to have bounded diameter, or weight them by the length of a core circle.

Here it would be useful to distinguish between answers none, finitely many, infinitely many, and if the third alternative, find some increasing function $f: \mathbb{R} \rightarrow$ $\mathbb{R}$ such that the ball of radius $r$ must contain at least $O(f(r))$ such components.

(2) Perhaps one should modify this question to measure generalized Reeb components. For example, the Whitehead manifold can be obtained as an increasing union of foliated "plugs" centered on a single Reeb component.

Question 5.2. What generalizations of the notion of taut foliation make sense on an open 3 -manifold? 
Remark. This question has different flavors in the topological and the geometric world.

(1) For $M$ the interior of a compact manifold with boundary, there are well-understood generalizations of a taut foliation or a taut sutured structure; see [41].

(2) If $M$ is an infinite cover of some closed $N$, and $N$ admits a vector field $X$ which is volume-preserving for some volume form on $N$, a foliation $\mathscr{F}$ of $M$ transverse to the cover $\widehat{X}$ probably has some useful properties. Maybe one should ask for $\mathscr{F}$ to be a Lipschitz section of $\widehat{X}$, in the sense that the transverse flow is uniformly bounded away from $T \mathscr{F}$. In this case, leaves of $\mathscr{F}$ are approximate minimal surfaces for some equivariant metric.

(3) One could weaken the transversality condition on $\widehat{X}$ to an asypmtotic or a statistical condition. For example: we could ask for every flowline of $\widehat{X}$ to be eventually transverse to $\mathscr{F}$, Or: assume $\mathscr{F}$ is co-oriented; then one could ask for every $\epsilon>0$ there should exist a $t$ such that for each flowline $\gamma$ of $\widehat{X}$, every segment of $\gamma$ of length $t$ is transverse to $\mathscr{F}$ in the positive direction.

(4) Rather than looking at a vector field $X$, one could ask for a single loop $\gamma$ in $N$ such that every lift $\widehat{\gamma}$ of $\gamma$ is transverse to $\mathscr{F}$ in the positive direction, and every leaf of $\mathscr{F}$ intersects some lift. Again, this could be made into an asymptotic or statistical condition.

\section{SUbLAMINATIONS AND SUPERLAMINATIONS}

\subsection{Essential laminations.}

Question 6.1. Characterize those essential laminations which contain genuine sublaminations.

Remark. Again, characterize can take several flavors. For example:

(1) Geometric conditions. For instance, if $\pi_{1}(M)$ is Gromov-hyperbolic, a lamination whose universal cover contains a quasi-geodesically embedded leaf contains a genuine sublamination.

(2) Topological conditions. For instance, if $\mathscr{F}$ is $\mathbb{R}$-covered or has one-sided branching, it cannot contain a genuine sublamination.

(3) Algorithmic conditions. Given a lamination, described somehow in finite terms, give an algorithm to determine whether it contains a genuine sublamination.

Given a branched surface $\mathscr{B}$, can the algorithm of Agol and Li can be modified to tell whether it carries (possibly not fully) a genuine lamination? (see [107] for related questions). The set of branched surfaces in a 3-manifold which fully carry an essential lamination can be constructed, so the problem reduces to showing, for two branched surfaces $\mathscr{B}, \mathscr{B}^{\prime}$ whether some splitting of $\mathscr{B}^{\prime}$ is carried by $\mathscr{B}$.

\subsection{Genuine laminations.}

Question 6.2. Suppose $\Lambda$ is a full genuine lamination; i.e. it has some complementary region which is an ideal polygon bundle over a circle. Suppose $M$ is hyperbolic. Is the core circle of this region isotopic to a geodesic? Does it have a noncoalescable insulator family?

Remark. (1) Noncoalescable insulator families are introduced in [44] where they are used to establish (virtual) topological rigidity of hyperbolic manifolds.

(2) Maybe bundle regions are exactly the wrong kind of region for this analogy. Does it work better if we ask whether cores of interstitial annuli of non-bundle regions 
have insulator families? After all, these regions look more like pared manifolds, where the annuli are like the pared locus, and therefore maybe have short geodesic representatives. Note that such interstitial annuli are unknotted in the universal cover, and therefore, if $\pi_{1}(M)$ is residually finite, in some finite cover they lift to circles isotopic to geodesics. See e.g. [40]

(3) Given some decomposition of $M-\Lambda$ into guts $\mathfrak{G}_{i}$ and interstitial $I$-bundles, the filling lemma from [22] says that $\Lambda$ can be filled to a lamination $\Lambda^{\prime}$ with gut regions isotopic to the gut regions of $\Lambda$, and for which every interstitial $I$-bundle is a product $I \times S^{1} \times \mathbb{R}^{+}$. Thus if $\gamma$ is the core of a gut region which is a polygon bundle over $S^{1}$, it is also the core of a complementary region (of a possibly different lamination) of an ideal polygon bundle over $S^{1}$. Perhaps such ideal polygon bundles are artificial; the more robust examples would be complementary regions to laminations with every leaf dense - i.e. minimal laminations.

Question 6.3 (Thurston). Suppose $\Lambda$ is a genuine lamination. When can $\Lambda$ be "filled in" to a very full lamination $\Lambda^{\prime}$ ? Does it help for $M$ to be hyperbolic?

Remark. (1) If $\Lambda^{\prime}$ is a co-oriented very full lamination, it can be filled in with monkey saddles to give a taut foliation. This implies for instance that closed leaves in any $\Lambda \subset \Lambda^{\prime}$ should be Thurston norm minimizing, and $\pi_{1}(M)$ should admit an action on a universal circle with no global fixed point. On the other hand, if we do not require $\Lambda^{\prime}$ to be co-oriented, there are no known homological obstructions. There are examples of homologically trivial closed surfaces which are leaves of very full laminations. (他)

(2) If $\Lambda$ is an essential lamination of $M$, then the universal cover $(\widetilde{M}, \widetilde{\Lambda})$ is topologically a product $\left(\mathbb{R}^{2}, \lambda\right) \times \mathbb{R}$ where $\lambda$ is a lamination of $\mathbb{R}^{2}$ (49]).

Since $\lambda$ is proper, there is a natural circular ordering on the ends of the leaves of $\lambda$, but this depends strongly on the (non-unique) choice of $\lambda$. If one can choose an equivariant representation $\left(\mathbb{R}^{2}, \lambda\right) \times \mathbb{R}$ of $(\widetilde{M}, \widetilde{\Lambda})$, one obtains a representation of $\pi_{1}(M)$ in Homeo $\left(S^{1}\right)$ with infinite image. It follows by [8] that $\pi_{1}(M)$ is isomorphic to a subgroup of $\operatorname{Homeo}\left(S^{1}\right)$. This happens for instance when $\Lambda$ is tight and has solid torus guts. See [22].

\subsection{Loosesse laminations.}

Definition 6.4. A lamination $\Lambda$ is loosesse if it satisfies all the properties of essentiality except possibly the no compressing monogons condition. This concept was introduced by Gabai.

Question 6.5 (Gabai). Are loosesse laminations good for anything? Are leaves of the universal cover of a loosesse lamination properly embedded? If $M$ contains a loosesse lamination, does $\widetilde{M}=\mathbb{R}^{3}$ ?

Remark. Brittenham has observed that for branched surfaces, the "no compressing monogons" condition can be substantially weakened to "no monogon bundles over $S^{1}$ ".

If $M$ contains a loosesse lamination $\Lambda$ for which $\widetilde{M}=\mathbb{R}^{3}$, is there an analogue of [49] to the effect that $(\widetilde{M}, \widetilde{\Lambda})$ is topologically $\left(\mathbb{R}^{2}, \lambda\right) \times \mathbb{R}$ for some (not necessarily proper) lamination $\lambda$ of $\mathbb{R}^{2}$ ?

Question 6.6. Give an example of a lamination in an atoroidal manifold - perhaps loosesse - which can never be realized by minimal surfaces for any metric, but which certifies some useful topological property of $M$. 
Remark. It is unknown whether every essential lamination can be realized by minimal surfaces for some metric; in some sense they seem to function as though they can be. But laminations containing compressing monogons can never be minimal surfaces for any metric.

\section{BRANCHED SURFACES AND TRIANGULATIONS}

Question 7.1 (Agol). Characterize branched surfaces embedded in 3-manifolds which can be non-trivially split to a homeomorphic copy of themselves.

Remark. (1) If $\mathscr{B}$ is such a branched surface, one can set $\mathscr{B}_{0}=\mathscr{B}$ and $\mathscr{B}_{i}$ the result of performing such a splitting on $\mathscr{B}_{i-1}$. If one fixes a triangulation $\tau$ transverse to $\mathscr{B}$, this procedure gives rise to a partial lamination $\mathscr{L}$ carried by $\mathscr{B}$ ("partial" because the injectivity radius of the subsurface which is being split open might be bounded above). If $\mathscr{B}$ carries a lamination, is $\mathscr{L}$ contained in some lamination $\Lambda$ carried by $\mathscr{B}$ ?

(2) If $\mathscr{B}$ supports a nontrivial transverse measure $\mu$, it supports a nontrivial measure projectively invariant under splitting (this follows from the fact that the set of nontrivial projective measures is a convex set, and the splitting operation induces a projective transformation of such a set, which necessarily has a fixed point). One can also look at nontrivial transverse measures twisted by some fixed holonomy representation of the fundamental group of $\mathscr{B}$. Is there some underlying geometric meaning of such measures? A basic example is a branched surface carrying the stable or unstable laminations of a pseudo-Anosov flow transverse to a surface bundle (such a branched surface can be derived from a sequence of splittings and collapses of an supporting train track).

Question 7.2 (Agol). Develop a theory of hierarchies for branched surfaces.

Remark. A branched surface $\mathscr{B}$ would be decomposed sequentially along train tracks into simple pieces. The idea would be to try to prove a gluing theorem about how essential laminations carried by the simple pieces of the hierarchy glue together to give essential laminations in the branched surface.

Something like this is done by $\mathrm{Li}$ in [80] for branched surfaces without sink disks.

Recall the notion of a taut ideal triangulation as introduced by Lackenby in [77], as a combinatorial refinement of certain ideal triangulations of hyperbolic 3-manifolds with torus cusps. Such objects give rise to branched surfaces which are very simple - their worst singularities look like train tracks $\times I$.

Question 7.3 (Dunfield). Which boundary slopes are realized by essential laminations carried by a fixed taut ideal triangulation? Give an algorithm.

Remark. Though these branched surfaces do not have sink disks, they have many half sink-disks, so not all of Li's constructions for manifolds with boundary can be made to work. These are good candidates for the "automatic laminations" in question 7.8 .

Question 7.4 (Schleimer). When does a Haken sum operation make sense for a pair of laminations in normal form with respect to a fixed triangulation?

Remark. For transversely measured laminations, this question is less interesting. Although, see Hatcher [68].

A prerequisite must be that the laminations intersect each tetrahedron in the same kind of quadrilateral pieces. This is not too far from the condition that they are simultaneously 
carried by a fixed branched surface. So a better question might be - what is the structure of the set of laminations carried by a given branched surface (up to monotone equivalence, say)? Is there a natural topology for which it is star-like? Or contractible?

7.1. Normal surfaces. The theory of normal surfaces was introduced by Haken in [64], although similar ideas were introduced in a more limited scope by Kneser. Any foliation can be normalized relative to some triangulation [7].

Question 7.5. Let $M$ be a 3-manifold, and $\Lambda$ an essential lamination. Let $C$ be a cycle representing the fundamental class of $M$. Is there a cycle $C^{\prime}$ with the same Gromov norm as $C$, and another essential lamination $\Lambda^{\prime}$ which is normal with respect to $C^{\prime}$ ?

Remark. (1) If $\Lambda$ is an incompressible surface, $C$ can be normalized by [2]. In fact, Agol points out in his paper that the same method works if $\Lambda$ is merely tight. Presumably, the cycle $C^{\prime}$ and the lamination $\Lambda^{\prime}$ will be derived from $C$ and $\Lambda$ by some process of straightening the simplices of $C$ relative to $\Lambda$, and compressing and evacuating (46]) $\Lambda$ with respect to its intersection with $C$.

A positive answer would extend Agol's volume bounds to manifolds with essential (not necessarily tight) laminations.

(2) Does it help to assume all the coefficients of $C$ are positive? In this case, for any singular simplex $\sigma: \Delta^{3} \rightarrow M$ in the support of $C$, and any leaf $\lambda$ of $\Lambda$ intersecting $\sigma\left(\Delta^{3}\right)$, we can develop (not uniquely) $C$ along any path $\gamma \subset \lambda$ which does not pass through any edges or vertices. For, once $\gamma$ comes to a face $\tau$ of $\sigma\left(\Delta^{3}\right)$, there is another singular simplex $\sigma^{\prime}: \Delta^{3} \rightarrow M$ whose boundary absorbs some of the mass of $\tau$; then $\Lambda$ can be developed along $\gamma$ into $\sigma^{\prime}$, and so on inductively. This gives a particular order in which to try to compress and evacuate the leaves of $\Lambda$ relative to $C$.

(3) If $C$ is a virtually embedded cycle - i.e. it comes from a triangulation in some finite cover $\widehat{M}, \Lambda$ can be lifted to $\widehat{\Lambda} \subset \widehat{M}$ and evacuated there. Can it be evacuated equivariantly?

Definition 7.6. A taut local orientation is a choice of ordering for the vertices of each tetrahedron in a triangulation with the following properties:

(1) The star of each vertex is ordered compatibly with a local foliation in normal form

(2) Every oriented loop is homotopically essential

Such objects are dual to branched surfaces with certain properties; in particular, certain examples give rise to branched surfaces which carry nothing, but fully carry essential laminations in finite covers. See [13] or [19].

Question 7.7. Suppose $\mathscr{B}$ is a branched surface in $M$ which is dual to a taut local orientation. Is there a finite cover of $M$ in which the pullback of $\mathscr{B}$ fully carries a lamination? What about an amenable cover?

Say an automatic lamination is given by the following data:

- A branched surface $\mathscr{B}$ with some fixed cell decomposition, so that there is a notion of combinatorial paths supported by this surface which can be enumerated.

- A lamination $\Lambda$ fully carried by $\mathscr{B}$.

- A finite state automaton A which recognizes combinatorial paths in $\mathscr{B}$ which represent geodesic paths in leaves of $\Lambda$. 
Remark. Note that if $\Lambda$ is essential and $M$ is atoroidal, leaves of $\Lambda$ are uniformly $\delta$ negatively curved, so such an automaton exists relative to an oracle which recognizes combinatorial paths in $\mathscr{B}$ which are carried by some leaf of $\Lambda$.

Question 7.8. Do branched surfaces without sink disks carry automatic laminations?

Remark. (1) This seems possible, insofar as Li's construction of a lamination carried by such a branched surface is local and explicit, and describes a procedure for recursively splitting open the branched surface. One subtle issue might be the choice of an extension over foliated circle bundles over punctured surfaces of positive genus: the existence of such an extension follows from the fact that every homeomorphism of $S^{1}$ is a commutator of length at most 2 . But it is not clear whether a recursive description of such a commutator can be given "automatically".

(2) Every time a combinatorial path passes over a source branch, there are two possible routes it can take. The sequence of splittings (positive, negative) so obtained is like a continued fraction expansion for an irrational number. The automaton $\mathrm{A}$ can only remember a bounded length of this sequence; this constrains the possible transverse structures supported by an automatic lamination. In particular, any automatic lamination should admit a natural transverse bilipshitz structure.

(3) This is related to question 8.7 on the existence of automatic left-orderings on finitely presented groups.

Question 7.9. Give a useful definition of thin position for an embedded graph $\Gamma \subset M$ with respect to a taut foliation $\mathscr{F}$. If $\Gamma$ is the $1-$ skeleton $\tau^{1}$ for a triangulation, can one find an isotopy such that the leaves of $\mathscr{F}$ are made up of polyhedral disks of bounded index?

Remark. For $\mathscr{F}$ a foliation by closed surfaces, one can make the leaves either normal or almost normal. If $\mathscr{F}$ is finite depth, one can normalize the depth 0 leaves. The depth 1 leaves are asymptotic to the depth 0 leaves, so they may be assumed to be normal away from a compact piece; some kind of relative minimal sweepout argument might produce an isotopy so that these leaves are almost normal. Continuing inductively, it seems that one can bound the index of the disks appearing in a leaf by a function of their depth. This argument should be made more precise.

\section{LEAF SPACES AND TRANSVERSE STRUCTURES}

The most significant nonexistence results in this area are the main theorems of [103] and [38] which give infinitely many examples of hyperbolic 3-manifolds $M$ for which there is no nontrivial action of $\pi_{1}(M)$ on a simply-connected 1-manifold, respectively order tree.

Question 8.1 (Thurston). Suppose $M$ is irreducible. Suppose further that $\pi_{1}(M)$ admits a nontrivial action on $\mathbb{R}$. When does $M$ admit a taut foliation with a transverse $\left(\pi_{1}(M), \mathbb{R}\right)$ structure?

Remark. (1) Given a representation $\rho: \pi_{1}(M) \rightarrow \operatorname{Homeo}(\mathbb{R})$, let $\left(E_{\rho}, \mathscr{F}_{\rho}\right) \rightarrow M$ be the associated foliated bundle. A section $s: M \rightarrow E_{\rho}$ transverse to $\mathscr{F}_{\rho}$ pulls back $\mathscr{F}_{\rho}$ to a foliation of $M$ with a transverse $\left(\pi_{1}(M), \mathbb{R}\right)$ structure.

(2) Given an action of a group $\Gamma$ on a simply connected 1-manifold $L$, when is there an immersion $i: L \rightarrow \mathbb{R}$ such that the action of $\Gamma$ descends to an action on $\mathbb{R}$ ? One should allow some flexibility in addressing this question, so that for instance one should be allowed to substitute monotone equivalent actions. Does it make a difference to weaken the quality of $i$ so that it is not necessarily an immersion, 
but merely (partial) order preserving? Under what circumstances is there a unique maximally ordered $\Gamma$-invariant order-preserving quotient $L \rightarrow L_{o}$ ? What if one imposes extra conditions on $\Gamma$, for instance that it should be amenable (especially if one allows monotone equivalent actions)?

(3) One can obviously generalize this question to actions on order trees.

Question 8.2. Suppose $\mathscr{F}$ is an $\mathbb{R}$-covered foliation of an atoroidal 3-manifold $M$. Is the holonomy representation $\rho_{H}$ of $\pi_{1}(M)$ on $\mathbb{R}$ conjugate to a group of coarse 1-quasiisometries? i.e. is there a positive valued function $C: \pi_{1}(M) \rightarrow \mathbb{R}$ such that

$$
\left|d\left(\rho_{H}(\alpha)(p), \rho_{H}(\alpha)(q)\right)-d(p, q)\right| \leq C(\alpha)
$$

for all $\alpha \in \pi_{1}(M)$ and $p, q \in \mathbb{R}$ ?

Remark. (1) If the value of $C$ can be chosen independent of $\alpha$, it follows that any two leaves of $\widetilde{F}$ are contained within bounded neighborhoods of each other. Such foliations are said to be uniform. All known examples of $\mathbb{R}$-covered but nonuniform foliations of atoroidal 3-manifolds satisfy the condition above.

(2) If $M$ is allowed to be toroidal, the action of $\pi_{1}(M)$ on $L$ can fail to satisfy the condition above. It is compatible with all known examples that for toroidal manifolds, the action of $\pi_{1}(M)$ should always be conjugate to a group of coarse quasiisometries.

See [117] and [12] for more details.

Question 8.3. Suppose $M$ is atoroidal and admits a taut foliation. Must it admit an $\mathbb{R}-$ covered foliation?

Remark. (1) There are many examples in [22] of small volume hyperbolic manifolds which admit essential laminations, but which do not admit $\mathbb{R}$-covered foliations. In fact, their fundamental groups admit no nontrivial representation in $\operatorname{Homeo}(\mathbb{R})$.

(2) If one is prepared to pass to a finite cover, one probably cannot find an example based on the nonexistence of group actions on certain 1-manifolds. For, with suitable homological hypotheses (which can be achieved by passing to a finite cover), the fundamental group of a tautly foliated manifold acts on $\mathbb{R}$ without a global fixed point. See [22] for details. On the other hand, [103] gives many examples of fundamental groups of hyperbolic 3-manifolds which admit no actions on $\mathbb{R}$ without a global fixed point, but which admit taut foliations and therefore have faithful circle actions. The Euler class of these circle actions are torsion. In general, for any 3 -manifold $M$ and for any 2-plane field $\xi$ on $M$ there is a 3 -manifold $N$ together with a map $f: N \rightarrow M$ that induces an isomorphism on homology (even over $\pi_{1}(M)$ ) such that $f$ is transverse to $\tau$, and such that the induced 2-plane field $f^{*} \xi$ on $N$ is homotopic to a taut foliation. For, Thurston's construction [113] lets us construct a foliation $\mathscr{F}$ on $M$ in a neighborhood of the 2-skeleton of an anti-oriented triangulation transverse to $\tau$. By drilling out spiralling paths, one can arrange for the complementary regions to the foliated region to be solid tori $S_{i}$. Then $N$ can be obtained from $M$ by drilling out these solid tori $S_{i}$ and filling in circle bundles $B_{i}$ over punctured surfaces. Any homeomorphism of $S^{1}$ can be realized as a commutator of bounded length, so the foliations of $\left.\mathscr{F}\right|_{\partial S_{i}}$ can be extended to foliations of $B_{i}$ transverse to the circle fibers. This gives a demonstrably taut foliation in the appropriate homology class on a manifold with the required properties. This construction is due to Thurston [119], and gives many examples 
of circle actions of fundamental groups of hyperbolic manifolds whose Euler class is torsion but non-zero.

(3) Brittenham, Naimi and Roberts ([11]) have produced examples of graph manifolds which admit taut foliations but do not admit $\mathbb{R}$-covered foliations.

(4) A related question from [45] is: if $M$ admits an essential lamination, does it admit a tight essential lamination? (an essential lamination is tight if the leaf space of the universal cover is Hausdorff). Quasigeodesic laminations in hyperbolic manifolds are tight, so a positive answer to question 10.4 would give the most positive answer to this question.

Question 8.4 (Agol). For a fixed manifold $M$, describe the structure of the set of all essential laminations with a transverse $S \widetilde{L(2, \mathbb{R})}$ structure.

Remark. (1) For a fixed triangulation $\tau$, the set of normal foliations supported by $\tau$ with a $S \widetilde{L(2, \mathbb{R})}$ structure can be algorithmically described, and has the natural structure of a semi-algebraic set. By Gabai [46], there is a fixed triangulation $\tau$ of $M$ so that every essential lamination of $M$ can be made normal with respect to $\tau$; in fact, there is a constructive procedure to find a finite set of branched surfaces which fully carry every nowhere dense essential lamination in $M$.

(2) One can study more generally the space of actions of $\pi_{1}(M)$ on $S L(2, \mathbb{R})$-trees, which one could ask to be either partially ordered or not. In this context, it is probably useful to differentiate between complete and incomplete trees. Any properly embedded interval $I$ in an $S L(2, \mathbb{R})$-tree, has a well-defined coarse length, a non-negative integer $l(I)$ which is the number of times $I$ wraps around $S^{1}$ under projection. An $S L(2, \mathbb{R})$-tree is complete if every properly embedded tight ray $r$ has infinite coarse length. In particular, if some element $\alpha$ stabilizes some point $p \in r$, it stabilizes infinitely many points on $r$ which exit the noncompact end of $r$. This problem is probably a good warm-up to understanding the space of actions of $\pi_{1}(M)$ on arbitrary order trees or 1-manifolds.

(3) Suppose $\Gamma$ acts on an $\mathbb{R}$ order tree $T$ in such a way that for every nontrivial $\gamma$, and for every properly embedded copy $l$ of $\mathbb{R}$ in $T$ invariant under $\gamma$, the set of fixed points of $\gamma$ is isolated, and is either empty or exits both noncompact ends of $l$, and the translation direction of $\gamma$ on the complementary intervals of $l$ alternate. Suppose furthermore that there is no sequence $\gamma_{i}$ with consecutive fixed points $p_{i}, q_{i}$ contained in a fixed segment $I$ for which $p_{i}, q_{i} \rightarrow r$ for some $r \in I$. Does $T$ carry the natural structure of an $S L(2, \mathbb{R})$-tree?

Question 8.5. Suppose $M$ admits a minimal taut foliation. What is the best analytic (transverse) quality of a taut foliation it admits? Can we find a minimal foliation such that the holonomy groupoid is of type $\mathrm{III}_{\lambda}$ for some algebraic $\lambda$ ? What about if one asks for a foliation monotone equivalent to the first? Homotopic?

Remark. There are well-known topological obstructions to smoothing transverse structures. [100] gives some important examples of foliations which can be made transversely $C^{1}$ but not $C^{2}$. At the level of Haefliger structures, the obstructions are related to homology of various groups of homeomorphisms of $\mathbb{R}^{n}$ ([62], [63], 120]).

A pseudogroup $\mathscr{G}$ of transformations of a measure space $(X, \mu)$ is said to be of type III $_{\lambda}$ if for each $g \in \mathscr{G}$, the Radon-Nikodym derivative $d\left(g_{*} \mu\right) / d \mu$ is well-defined on a set of full measure and takes values in $\langle\lambda\rangle$, the abelian group of powers of $\lambda$. Such measures and actions arise frequently from automatic structures; the theory of Patterson-Sullivan measures is also relevant. 
An obvious obstruction is that $\pi_{1}(M)$ must act on some 1-manifold with this quality. Question 7.8 and question 8.10 are relevant here. See [29] for an abstract discussion of transverse measure theory for foliated manifolds.

Question 8.6. Is there a universal constant c such that a hyperbolic 3-manifold $M$ whose fundamental group $\pi_{1}(M)$ can be ordered out to radius c can be left-ordered? Or weaker, is there an effective method to compute such a $c(M)$ for a given $M$ ?

Remark. (1) A 3-manifold group $\pi_{1}(M)$ is left orderable iff it admits a faithful representation in $\mathrm{Homeo}^{+}(\mathbb{R})$. If $M$ admits an $\mathbb{R}$-covered foliation, the representation $\pi_{1}(M) \rightarrow \operatorname{Homeo}(L)$ may not be faithful, but the kernel is a surface group, and is itself left-orderable, so the representation is monotone equivalent to a faithful one.

(2) The set of finitely presented groups which are not left-orderable is recursively enumerable; on the other hand, the set of finitely presented groups which are leftorderable is not. Does hyperbolic geometry, or more generally word-hyperbolic geometry make a difference here? A word-hyperbolic group looks more or less free on a large scale; free groups are certainly left-orderable. A positive answer to this question would give an algorithm to detect left-orderability (of hyperbolic 3-manifold groups). In fact, any $M$ which admits a taut foliation, a pseudoAnosov flow, or certain kinds of genuine laminations, has a faithful representation $\pi_{1}(M) \rightarrow \operatorname{Homeo}\left(S^{1}\right)$ coming from a universal circle; if $M$ is a rational homology sphere, this representation lifts to $\left.\widetilde{\operatorname{Homeo}(} S^{1}\right)$ when restricted to the commutator subgroup, a finite index subgroup. Such periodic representations might be easier to detect or rule out than arbitrary ones. In particular, in [22] it is proved that the Weeks manifold does not admit a pseudo-Anosov flow, or a tight essential lamination. On the other hand, there are examples of 3-manifolds which act faithfully on $S^{1}$ but not on $\mathbb{R}$. See [22] for more details.

Question 8.7. Let $\mathrm{T}$ be some class of abstract computers; e.g. finite state automata, Turing machines, Turing machines relative to some oracle $O$, etc. A T-order on a group $G$ is a left-invariant order such that there is a machine $T \in \mathrm{T}$ which recognizes the positive cone $G^{+} \subset G$. What kinds of $\mathrm{T}$-orders are possible for fundamental groups $G$ of hyperbolic 3-manifolds?

Remark. (1) A special case consists of the class $T$ of finite state automata. One can ask whether there exists an automatic order on $G$; i.e. whether there is an FSA which can detect geodesic words with values in the positive cone. Of course, $G$ must already be an automatic group for there to be any chance at all. It turns out that this class of groups is very small. There are automatic orders on $\mathbb{Z}^{n}$ for all positive integers $n$, but there is no automatic order on $F_{2}$, the free group on two generators. A proof is as follows. Let $F_{2}=\langle a, b\rangle$ be a generating set. A leftinvariant order on $F_{2}$ is more or less equivalent to a faithful action of $F_{2}$ on $\mathbb{R}$ by orientation-preserving homeomorphisms. We may also assume this action is minimal. Let 0 be a point with trivial stabilizer, so that the positive cone are the elements $g$ with $g(0)>0$.

Suppose $w$ acts as a positive translation on a $w$-invariant interval $I$, with lowest point $p<0$ and highest point $p^{\prime}>0$. Since the action is minimal, there is $q \in I$ and a word $v$ such that $v(q)=p^{\prime}$. Let $x_{1}$ be a word with $x_{1}(0)=p_{1}^{+}$, where 
$p_{1}^{+}>p$ is very close to $p$. Then

$$
w^{-n} v w^{n} x_{1}(0)>0
$$

for $n>N_{1}$, and

$$
w^{-n} v w^{n} x_{1}(0)<0
$$

for $n \leq N_{1}$ for some very large $N_{1}$. We can choose $x_{i}$ with $x_{i}(0) \rightarrow p$ for which there are corresponding $N_{i} \rightarrow \infty$. If $\mathrm{A}$ is a fixed finite state automaton, eventually $N_{i}$ exceeds the memory capacity of $\mathrm{A}$, and $\mathrm{A}$ is incapable of distinguishing $w^{-N_{i}} v w^{N_{i}} x_{i}$ from $w^{-N_{i}-1} v w^{N_{i}+1} x_{i}$.

Conversely, if every $w$ fixes at most 1 point on $\mathbb{R}$, the image of the group is solvable, and therefore not faithful.

Since every hyperbolic 3 -manifold group contains a quasigeodesically embedded $F_{2}$, this implies that no hyperbolic 3 -manifold group admits an automatic order.

(2) If a finitely generated automatic group $G$ is left-orderable, given a lexicographic ordering on representatives of $G$ there is a well-defined lexicographically first left-order. What is the algorithmic quality of this order? Note that given an automatic group $G$, there is an algorithm which either certifies that $G$ is not leftorderable, or recursively enumerates the words in the lexicographically first leftorder.

(3) The general question of whether or not a finitely presented group is left-orderable is undecidable. But even if a group is left-orderable, it might be much harder to describe an explicit left-order than merely to certify orderability.

\subsection{Universal circles.}

Question 8.8. Let $\Lambda^{ \pm}$be a pair of laminations of $S^{1}$ which are transverse to each other and have finite area complementary domains. Suppose $\Gamma$ is a group of automorphisms of $S^{1}$ which preserves $\Lambda^{ \pm}$and acts minimally on the leaves of either lamination. When is $\Gamma$ commensurable with $\pi_{1}(M)$ for $M$ a hyperbolic 3-manifold?

Remark. (1) If $\Gamma=\pi_{1}(M)$ for some $M$, must $M$ admit a pseudo-Anosov flow $X$ for which the associated action on the universal circle of $X$ is conjugate to the given action of $\pi_{1}(M)$ on $S^{1}$ ? Actions of 3-manifold groups on circles associated to pseudo-Anosov flows are constructed in [22].

(2) What properties should a lamination $\Lambda$ or collection of laminations of $S^{1}$ satisfy to ensure good geometric control over the subgroup of $\operatorname{Homeo}\left(S^{1}\right)$ preserving $\Lambda$ ? For instance, let $\Lambda^{ \pm}$be as above and $\Gamma$ a group of automorphisms of $\Lambda^{ \pm}$: is $\Gamma$ word hyperbolic? Is there a natural topology on the space of such pairs $\Lambda^{ \pm}$for which a large group $\Gamma$ of automorphisms exists? What do non-trivial convergent sequences (of commensurability classes of hyperbolic 3-manifolds) look like in such a topology?

(3) Given a pair of laminations $\Lambda^{ \pm}$of $S^{1}$ as above, the natural quotient of $S^{1}$ by the leaf relations of $\Lambda^{ \pm}$is topologically $S^{2}$. If $\Gamma$ is a group of automorphisms of $\Lambda^{ \pm}$, we get a representation $\Gamma \rightarrow \operatorname{Homeo}\left(S^{2}\right)$ and a flat bundle over a $B \Gamma$. The topological group $\operatorname{Homeo}\left(S^{2}\right)$ is homotopy equivalent to $O(3, \mathbb{R})$ by Smale; are the cohomology classes pulled back via the associated classifying map $B \Gamma \rightarrow$ $B O(3, \mathbb{R})$ bounded in norm in terms of combinatorial data that can be read off from $\Lambda^{ \pm}$? 
Question 8.9. What possibilities are there for universal circles $S_{\text {univ }}^{1}$ for a fixed manifold? For a fixed foliation? For what taut foliations is there a unique minimal universal circle?

Remark. (1) A universal circle $S_{\text {univ }}^{1}$ for a taut foliation $\mathscr{F}$ formally gives rise to a nontrivial invariant lamination of $S_{\text {univ }}^{1}$ for each direction in which $\widetilde{\mathscr{F}}$ branches ([20]). Given an action of a group $G$ on a circle $S^{1}$ preserving a lamination $\Lambda$, one can modify the action in quite drastic ways: one can take an orbit class of complementary region, and "flip" the lamination along every edge of every region in the orbit. If $T$ is the dual planar tree to $\Lambda$, this is equivalent to re-embedding $T$ in the plane in such a way that the circular order is reversed at every vertex in an orbit class (see [22]). If there is more than one orbit class of complementary region, this operation is not a topological conjugacy, and the circle actions in question are quite distinct. One can almost certainly use this operation to produce examples of minimal circle actions of hyperbolic 3-manifold groups which are not universal circles for any foliations.

(2) There is a unique minimal universal circle for $\mathscr{F}$ an $\mathbb{R}$-covered foliation, or one with one-sided branching. These minimal universal circles are rigid under certain kinds of deformations of the foliation, in many cases.

(3) The best answer would do two things: firstly it would give a good description of the "representation space" of $\pi_{1}(M)$ in $\operatorname{Homeo}\left(S^{1}\right)$ and secondly it would explicitly characterize the subspace of representations of geometric origin.

It is probably unreasonable to expect an algebraic structure on the representation space, but a polyhedral structure might be more promising, by analogy with question 3.1. A sequence of isotopies of a fixed pseudo-Anosov flow degenerating to a flow corresponding to a higher codimension face should give rise to a monotone relation between the universal circles of the leaf space of the flows.

(4) Universal circles for certain kinds of genuine laminations and for pseudo-Anosov flows are constructed in [22]. Perhaps every essential lamination gives rise to a universal circle.

Question 8.10. What is the best analytic quality for the action of $\pi_{1}(M)$ on a universal circle $S_{\text {univ }}^{1}$ ?

Remark. (1) It is probably unreasonable to expect the action to be $C^{2}$ if $M$ is hyperbolic. On the other hand, any minimal action of a finitely generated group on $S^{1}$ is conjugate to a Lipschitz action, with respect to a harmonic measure. For an introduction to the rich panorama of subgroups of homeomorphisms of $S^{1}$ stretching between Lipschitz and $C^{2}$, see [122].

(2) The following related question was suggested by Barry Mazur. Let $G$ be a finitely presented group, and let $X, Y$ be compact smooth manifolds with $\operatorname{dim}(X)<$ $\operatorname{dim}(Y)$. Suppose $\rho_{X}: G \rightarrow \operatorname{Homeo}(X)$ and $\rho_{Y}: G \rightarrow \operatorname{Homeo}(Y)$ are minimal actions such that there is a surjection $f: X \rightarrow Y$ which intertwines $\rho_{X}$ and $\rho_{Y}$. When are $\rho_{X}, \rho_{Y}$ actions by diffeomorphisms for some appropriate smooth structures on $X, Y$, and how can one find construct such a smooth structure on $X$ from a smooth structure on $Y$ and from $f$ ? This question is most interesting when $X$ is a circle or an interval, but there are probably many interesting examples of $Y$. What seems plausible is that high dimensional cohomology of $G$ pulled back from the action on $Y$ represents an obstruction to stiffening the analytic quality of the action on $X$. 


\section{Classical 3-MANifold theory}

Question 9.1. Is there a universal transverse surgery description of tautly foliated manifolds, in the sense that there is a fixed $M$ such that for every tautly foliated manifold $N, \mathscr{F}$ there is a link $L \subset N$ transverse to $\mathscr{F}$ so that $M$ is obtained from $N$ by surgery on $L$ ?

Remark. (1) The idea is to get an analogue of Lickorish's theorem (that every closed 3 -manifold is surgery on a link in $S^{3}$ ) in the foliated context. One should also have in mind Thurston's observation (see [1]) that the process of repeatedly drilling out the shortest geodesics in a sequence of hyperbolic manifolds tends to stabilize to a "universal" sequence. Question 10.5 is probably relevant to an approach along these lines. One should concentrate on taut foliations for this question, so that there are enough transverse knots to "see" the whole topology of $N$. One idea might be to start with a triangulation $\tau$ in normal form with respect to $\mathscr{F}$, and look at the interaction of the associated Heegaard decomposition with $\mathscr{F}$.

(2) We can modify the question to ask whether, given two tautly foliated manifolds $M_{1}, \mathscr{F}_{1}$ and $M_{2}, \mathscr{F}_{2}$, there are links $L_{i}$ transverse to $\mathscr{F}_{i}$ such that $M_{1} \backslash L_{1}=$ $M_{2} \backslash L_{2}$.

Question 9.2. Give a collection of fundamental operations on foliations and an explicit family of base foliations such that every tautly foliated manifold $M, \mathscr{F}$ is obtained from one of the base family by repeated application of fundamental operations.

Remark. (1) It is unclear if even the following naive list of operations

- branched covers and pushforward

- cut-and-shear

- torus connect sum

- tangential surgery

- surface bundle plumbing along transverse train-tracks

- monotone deformations

- cut-and-paste furrows along loops with linear holonomy

and base foliations

- foliations of circle bundles

- transversely measured foliations

- finite depth foliations and those representing irrational rays in $\mathrm{H}_{2}$

- foliations derived from alternating knots by Delman-Roberts methods

would fail to suffice. The idea is really to develop some more robust invariants of foliations which vary either not at all, or in some well-understood way under basic operations, and at the same time to give a procedure for recognizing orphans with respect to a given list of operations.

(2) A model theorem along these lines is the recent result of Noah Goodman and, independently, Giroux, improving work of Gabai, which says that the set of fibered links in a homology 3-sphere $M$ are related by a sequence of elementary stabilizations and destabilizations, where the stabilization operation is plumbing with a Hopf band.

\subsection{Persistence questions.}

Question 9.3. What is the most general class of knots to which the techniques of DelmanRoberts (in constructing persistent laminations) can be extended?

Remark. The main reference is [30]. 
The idea of this question is that the lamination should be constructed in an essentially algorithmic way from a projection of the knot. A related question asks what information essential laminations can give about projections of knots; for instance, when can foliated techniques be used to certify that a knot projection has minimal number of crossings? Is the problem of minimal crossing number for knot projection NP-complete?

Question 9.4 (Delman). Suppose $K$ is a non-torus alternating knot. Then essential laminations can be constructed which realize every (nontrivial) boundary slope. Can essential laminations be constructed with an even sided bundle complementary region containing $K$, so that every nontrivial surgery can be filled in with a monkey saddle?

\subsection{Distance of Heegaard splittings.}

Question 9.5. It is known [66] that if a 3-manifold $M$ contains an essential surface of genus $g$, the distance of any Heegaard splitting of $M$ has distance at most $2 g$. Does the "distance filtration" put any useful structure on the essential laminations supported by a given $M$ ? i.e. if $M$ admits Heegaard splittings of distance at least $2 g$, what can one say about the essential laminations $\Lambda$ contained in $M$ ?

Remark. A preliminary technical issue would be to decide what a tight intersection of $\Lambda$ with the splitting surface $\Sigma$ should be, and then try to tighten any given $\Lambda$ with respect to $\Sigma$, perhaps by means of an infinite process, by analogy with Brittenham's principle (see [10] and [46]).

\section{HYPERBOLIC GEOMETRY}

\subsection{Asymptotic geometry of leaves.}

Question 10.1. Suppose $\mathscr{F}$ is a taut foliation of a hyperbolic 3-manifold $M$ with twosided branching. Must there be a leaf $\lambda$ of $\widetilde{\mathscr{F}}$ whose complement contains an open halfspace of $\mathbb{H}^{3}$ on either side? We call such a leaf asymptotically separated.

Remark. This question is equivalent to the condition that the limit set of some leaf of $\widetilde{F}$ is not all of $S_{\infty}^{2}$. A conjecture of Fenley says that $\lambda_{\infty}=S_{\infty}^{2}$ for some leaf $\lambda$ of $\widetilde{F}$ iff $\mathscr{F}$ is $\mathbb{R}$-covered.

Question 10.2. Do leaves of $\widetilde{\Lambda}$ for $\Lambda$ an essential lamination have the continuous extension property? More generally, what is the relationship between the action of $\pi_{1}(M)$ on various ideal boundaries of $\widetilde{M}$ arising from the foliated structure (e.g. universal circles) and the ideal boundaries arising from the geometry of $\widetilde{M}$.

Remark. (1) The seminal result in this area is the theorem of Cannon and Thurston [27] that leaves of surface bundles over $S^{1}$ extend continuously to sphere filling curves. The method of Cannon and Thurston has been greatly extended and improved by Fenley to deal with many (most?) finite depth foliations, and certain other taut foliations [34, 35, 36].

(2) A measurable extension property is much easier to establish, and follows from exponential growth of hyperbolic spaces, and the fact that a leaf of an essential lamination in the universal cover is uniformly properly embedded in its $\epsilon$ neighborhood for some $\epsilon$. See [17]. Note that even in cases where the continuous extension property is known leafwise (e.g. finite depth foliations) it is not known for all or any universal circles (although Fenley has some unpublished partial results on this question). 
Question 10.3. Suppose $\mathscr{F}$ is a finite depth foliation of a hyperbolic 3-manifold. What is the relationship (if any) between the Hausdorff dimension of the limit set of a leaf $\lambda$ of $\widetilde{F}$ and the depth of $\mathscr{F}$ or $\lambda$ ?

Remark. (1) Conjecturally, a foliation is $\mathbb{R}$-covered iff the limit set of any (and therefore every) leaf is all of $S_{\infty}^{2}$. A proper foliation of depth $>0$ contains a quasigeodesic leaf; in particular, depth 0 is distinguished from any other finite depth by the property that the limit set of every leaf has Hausdorff dimension 2. More generally, if $M^{\prime} \subset M$ is the union of the leaves of highest depth, the components of $\widetilde{M}_{\infty}^{\prime}$ and $\lambda_{\infty}$ for leaves $\lambda$ of highest depth should agree.

(2) For depth one leaves, the components of $M^{\prime}$ are the interiors of compact hyperbolic manifolds with quasigeodesic boundary. For such manifolds, elements of $\pi_{1}$ can be enumerated by a finite state automaton. Does this mean that the Hausdorff dimension of the limit set is a special value of an $L$-function?

(3) One can numerically estimate the Hausdorff dimension of the limit set of a noncompact leaf, using a harmonic measure for the universal circle to determine which directions at infinity (in the intrinsic geometry of the leaf) are most distorted in the ambient geometry. This should give a fast numerical algorithm to tell whether or not a foliation of a hyperbolic manifold is of depth one. On the other hand, when $M$ has cusps, there are numerical problems with this approach, since it is slow to enumerate elements of $\pi_{1}(M)$ which exit a cusp. This is especially troubling, since the case one would be most interested in is foliations of knot or link complements. It is an interesting problem to find therefore a fast numerical algorithm to estimate the Hausdorff dimension of the limit set of a leaf in a foliation of a cusped manifold. Obviously, the foliation itself must be described in some computationally effective manner. Foliations carried by Lackenby's taut ideal triangulations [77] are good candidates for study.

\subsection{Realization questions.}

Question 10.4 (Thurston). Suppose $M$ an atoroidal 3-manifold admits an essential lamination. Does it admit a (necessarily genuine) lamination with quasi-geodesic leaves?

Remark. (1) Any atoroidal manifold with an essential lamination contains a genuine lamination $\Lambda$, and therefore has word-hyperbolic fundamental group ([20], [50]). If such a lamination is not quasi-geodesic, perhaps one can find another (more quasi-geodesic?) lamination transverse to it which is an "eigenlamination" for the extrinsic distortion of the geometry of $\widetilde{\Lambda}$. As a caveat, one should note that in general there is no naive notion of a universal circle for a genuine lamination, so some new ideas are necessary. Note that faithful circle actions are associated to certain essential laminations in [22], but these do not give monotone relations between circles at infinity of leaves, as a real universal circle should. Rather they function as a kind of circular parameterization of the leaf space of the laminations. Moreover, even such naive circular parameterizations do not exist for certain nontight genuine laminations.

(2) A lamination with quasi-geodesic leaves is tight, so a first step would be to try to replace a non-tight lamination with a tight one. Associated to a cataclysm (i.e. a collection $\left\{\mu_{i}\right\}$ of incomparable non-separated leaves of $\widetilde{\Lambda}$ which are the limit of a comparable monotone sequence) in a non-tight lamination $\Lambda$, there is a geodesic lamination of $\mathbb{H}^{2}$ whose complementary regions parameterize the $\mu_{i}$. 
(3) One reason to be interested in the existence of such a lamination is that it would be an important technical advantage in Thurston's program to geometrize laminar 3 -manifolds. Given a choice of conformal structure on the each pair

$$
\text { (complementary region } C \text {, boundary leaf of } C \text { ) }
$$

leaves of $\Lambda$, one can try to generalize the skinning map as a map from Teich $\left(\bigcup_{C} \partial C\right)$ to itself. In the case of a taut foliation, it might proceed as follows. A universal circle gives a monotone relation between the circles at infinity of the leaves of $\widetilde{\mathscr{F}}$. In fact, for $\lambda_{i}$ a maximal collection of incomparable leaves above or below $\mu$, for any leaf $\mu$ of $\widetilde{\mathscr{F}}$, there is a monotone relation between $S_{\infty}^{1}(\mu)$ and a "cactus" made from the the union $\bigcup_{i} S_{\infty}^{1}\left(\lambda_{i}\right)$. For each leaf $\lambda$ of $\widetilde{\mathscr{F}}$, we should choose a pair of points

$$
\tau^{ \pm}(\lambda) \in \operatorname{Teich}\left(D^{2}\right) \times \operatorname{Teich}\left(\overline{D^{2}}\right)
$$

a product of universal Teichmüller spaces as an initial guess. Then monotone relations between circles at infinity give a new marking $\sigma\left(\tau^{+}\right)$obtained by sewing together the markings $\tau^{+}\left(\lambda_{i}\right)$ by the monotone relation. There are numerous problems with such a program; for instance, there are many possibilities for leaves $\lambda_{i}$; moreover, there is no natural decomposition of a laminated manifold along a collection of leaves into a compact "fundamental domain", especially if $\mathscr{F}$ is minimal. Secondly, the domain obtained by gluing together copies of $\tau^{+}\left(\lambda_{i}\right)$ will typically not be a quasidisk, but will be pinched along an infinite lamination. One can try to remedy this problem by letting $\sigma\left(\tau^{+}\right)$be equal, not to the union of the $\tau^{+}\left(\lambda_{i}\right)$ sewn together, but by $\tau^{+}$bent in the direction of this pinching lamination. But more importantly, no foliation of a hyperbolic manifold can have all leaves quasi-geodesically embedded; in particular, for all but the most trivial cases, there should be no fixed point for the skinning map in $\left(\operatorname{Teich}\left(D^{2}\right) \times \operatorname{Teich}\left(\overline{D^{2}}\right)\right)^{L}$, but rather in some natural compactification, analogous to the Thurston boundary of Teichmüller space.

For a quasigeodesic lamination, at least a fixed point for the skinning map should exist in some universal Teichmüller space, and proving geometrization in this case might be considerably easier.

\subsection{Interactions of foliations with hyperbolic structures.}

Question 10.5. What do short geodesics look like with respect to taut foliations? Is there a universal $\epsilon$ such that for every hyperbolic manifold $M$, every taut foliation $\mathscr{F}$ of $M$, and every geodesic $\gamma$ with $|\gamma|<\epsilon$, $\gamma$ is either isotopic into a leaf of $\mathscr{F}$ or isotopic to be transverse to $\mathscr{F}$ ? What about homotopic?

Remark. (1) For a fixed taut foliation $\mathscr{F}$, leaves of $\widetilde{\mathscr{F}}$ are uniformly properly embedded in their $\epsilon$-neighborhoods, for some uniform $\epsilon$. If $\mathscr{F}$ does not have 2-sided branching, every loop is homotopic to either a transverse or a tangential loop; this question asks whether branching of $\widetilde{\mathscr{F}}$ takes place on a scale uniformly bounded below over all taut foliations.

(2) For $M$ a hyperbolic manifold with a short geodesic $\gamma$, it is an empirical observation of Rubinstein, Jaco and Sedgewick that a 1-efficient triangulation (see [72]) has a "layered" solid torus corresponding to $\gamma$, which has a very standard combinatorial form. We can try to normalize $\mathscr{F}$ relative to such a triangulation, possibly evacuating a subfoliation in the process $([46])$. Can one use the explicit combinatorial structure of the layered solid torus to control the interaction of $\gamma$ with $\mathscr{F}$ ? 
Question 10.6. Is there a uniform bound on the Godbillon-Vey invariants of the taut foliations of a hyperbolic manifold in terms of its volume?

Remark. Some estimate for foliations on fixed $M$ can be obtained from the theorem of Gabai [46] that there is a fixed triangulation $\tau$ of $M$ such that every taut foliation of $M$ can be made normal with respect to $\tau$. On the other hand, there are no known a priori bounds on how many simplices such a $\tau$ must have in terms of the number of simplices in a minimal triangulation, or what relation $\tau$ has to the geometry of $M$. For instance, there are geodesic triangulations which cannot be made "virtually transverse" to certain taut foliations; i.e. there is no finite cover in which the pullback of the triangulation can be normalized relative to the pullback of the foliation.

Question 10.7. Suppose $\mathscr{F}$ is a taut foliation of a hyperbolic 3-manifold M. Let

$$
\pi: \widetilde{M} \rightarrow L
$$

be the projection to the leaf space of $\widetilde{\mathscr{F}}$.

(1) For $\gamma$ a random walk in $\widetilde{M}$ (which is isometric to $\mathbb{H}^{3}$ ), what is the typical behaviour of $\pi(\gamma)$ ?

(2) Does a random walk in $\widetilde{M}$ converge to a definite end of $L$ ?

(3) What if we replace "random walk" with "random geodesic" in the previous question?

(4) Is the pushforward of asymptotic behaviour well-defined? That is, is it true that for a set of geodesics $\gamma$ of full measure, for all $\gamma^{\prime}$ a bounded distance from some $\gamma$ the behaviour of the pushforward of $\gamma$ and $\gamma^{\prime}$ exit the same end of $L$ ?

(5) Suppose $\mathscr{F}$ has one-sided branching. Does a random walk always exit $L$ in the unbranching direction?

(6) Suppose there is a positive probability of a random walk exiting a proper positive or negative end of $L$. Does this imply $\mathscr{F}$ is $\mathbb{R}$-covered? What about if there is a positive probability of a random walk being recurrent?

Remark. (1) For the basic theory of random walks and Brownian motion, see e.g. [108].

(2) For $\mathscr{F}$ transversely measured, $L$ is naturally isomorphic to $\mathbb{R}$. Then the pushforward of a random walk or geodesic on $\widetilde{M}$ under $\pi$ is a random walk on $\mathbb{R}$, and in particular the pushforward is recurrent.

(3) The question about foliations with one-sided branching has a positive answer if the limit set of some (and therefore every) leaf of $\widetilde{\mathscr{F}}$ is not all of $S_{\infty}^{2}$. For in this case, the limit set of any leaf is a dendrite of measure 0 , and the complement is entirely on the nonbranching side of the leaf.

(4) One has to be very careful in defining the behaviour of a random walk on $L$; coarsely equivalent models for random walks on $\widetilde{M}$ (e.g. combinatorial, piecewise geodesic, etc.) might give rise to very different behaviour in $L$. For instance, for many $\mathscr{F}$ with $2-$ sided branching, we can find a pair of proper lines $\gamma_{1}, \gamma_{2}$ in $\widetilde{M}$ which are asymptotic (i.e. the distance between them converges to 0 ) but for which $\gamma_{1}$ exits an end of $L$ and $\gamma_{2}$ is contained in a leaf. On the other hand, if $\gamma_{1}, \gamma_{2}$ are a bounded distance apart, and $\gamma_{1}$ exits to an incomparable end of $L$ (i.e. the limit of a sequence of leaves $x_{1}>y_{1}<x_{2}>y_{2}<x_{3}>y_{3}<\ldots$ where the $x_{i}$ are all incomparable and the $y_{i}$ are all incomparable) then $\gamma_{2}$ exits to the same incomparable end. Moreover, if $\mathscr{F}$ arises from a slithering or branched slithering, if $\gamma_{1}$ exits any end, $\gamma_{2}$ a bounded distance away exits the same end. If $\pi(\gamma)$ exits 
an end of $L$, with probability one does it exit an incomparable end? (Yes in many cases)

(5) If $\mathscr{F}$ is finite depth, we can "trap" the behaviour of a random walk by compact leaves. If $\mathscr{F}$ is not a surface bundle over a circle, the random walk has a definite probability of making an incomparable detour in a fixed time interval. The tree of incomparable detours branches with ramification bounded below; so a random walk will exit an incomparable end with probability 1 in this case. Probably the same result is true if $\widetilde{\mathscr{F}}$ contains any leaf which is asymptotically separated. Conjecturally, every leaf of every $\mathscr{F}$ with 2 -sided branching should be asymptotically separated. However, part of the motivation for studying this question would be to address this conjecture, rather than the other way around.

Question 10.8. Suppose $\Lambda$ is an essential lamination of a hyperbolic manifold $M$. Is $\Lambda$ isotopic to a lamination whose curvature is bounded below everywhere by -2 ?

Remark. A complete properly embedded minimal surface in $\mathbb{H}^{3}$ has curvature bounded below by -2 everywhere. Also see question 4.1 .

\section{Foliated TEICHMÜlLER THEORY}

11.1. Deformations, mapping-class groups. For $\mathscr{F}$ a taut foliation of an atoroidal $3-$ manifold, any conformal class of leafwise metric on $\mathscr{F}$ can be "uniformized" to a leafwise hyperbolic metric. Co-deformations of such metrics are parameterized by leafwise quadratic holomorphic differentials, and presumably analogues of many other elements of one-dimensional complex analysis can be found.

Question 11.1. What kind of nontrivial "mapping class elements" are possible for taut foliations?

Remark. (1) If $\mathscr{F}$ is a foliation of an atoroidal manifold $M$ arising from a slithering over $S^{1}$, there is a canonical (up to isotopy) transverse pseudo-Anosov flow $X$ associated to the slithering. This flow is not in general leaf preserving at all times, but the time one flow takes $\mathscr{F}$ to itself, and qualifies as a "mapping class element" of infinite order. Slitherings, and slightly more generally, uniform foliations are the only foliations for which the time one flow of a transverse vector field takes leaves to leaves; are all such mapping class elements isotopic to the canonical automorphism?

(2) For arbitrary taut $\mathscr{F}$ and atoroidal $M$, the mapping class group of $M$ is finite, by results of [20] and [51]. If $\phi$ is a mapping class of $M, \mathscr{F}$ of infinite order, for some finite power $\phi$ will be isotopic to the identity. Lift $\phi$ to $\widetilde{\phi}: \widetilde{M} \rightarrow \widetilde{M}$. It follows that there is a universal $t$ such that for every leaf $\lambda$ of $\widetilde{F}$, the Hausdorff distance between $\lambda$ and $\widetilde{\phi}(\lambda)$ is $\leq t$. In particular, the space of leaves between $\lambda$ and $\widetilde{\phi}(\lambda)$ is isotopic to a product, and so if $\mathscr{F}$ branches, every leaf corresponding to a non-Hausdorff point of $L$ is fixed by $\widetilde{\phi}$. For example, if $\mathscr{F}$ is finite depth, the only leaves which can move are those of highest depth. If $\mathscr{F}$ is $\mathbb{R}$-covered but not uniform, there is a monotone equivalent foliation $\mathscr{G}$ to which an element homotopic to $\phi$ descends, which fixes $\widetilde{\mathscr{G}}$ leafwise.

\subsection{Transverse Teichmüller flows.}

Question 11.2. A foliation is taut iff it admits a volume-preserving transverse flow. PseudoAnosov flows are good candidates for "best" such transverse flows, when they exist, which 
is frequently. Is there an analytic construction of pseudo-Anosov flows, by analogy with Bers' proof of Thurston's classification of surface automorphisms?

Remark. Fix a conformal sturcture on $\mathscr{F}$, and let $C$ be a transverse cone field. Amongst unit speed vector fields $X$ transverse to $\mathscr{F}$, there are quasiconformally extremal ones, by a standard compactness argument. It is not a priori clear that there should be an extremal vector field amongst all transverse unit speed vector fields. Moreover, one would want a "best" flow to be metric independent. If $\mathscr{F}$ arises from a slithering, it makes sense to parameterize a transverse vector field so that the time 1 flow is a unit of the slithering; the dilatation of the time 1 map is well-defined with respect to a leafwise conformal structure. Of course, one must simultaneously optimize over all transverse flows and all choices of leafwise conformal structure.

For more general foliations, one needs to decide how to naturally parameterize the leafwise flow in order to measure (infinitesimal) dilatation. One plausible choice is to measure the result of flow with (transverse) speed equal to the magnitude of a harmonic transverse measure.

Question 11.3. Suppose $M$ is atoroidal and $\mathscr{F}$ arises from a slithering over $S^{1}$. Let $X$ be pseudo-Anosov transverse to $\mathscr{F}$, such that the time 1 flow $Z$ takes $\mathscr{F}$ to itself. Lift to $\widetilde{M}$ and let $\lambda, Z^{n}(\lambda)$ be leaves of $\widetilde{\mathscr{F}}$, both uniformized as $\mathbb{H}^{2}$ by Candel's theorem. Can $Z$ be approximated by mapping class elements between compact surfaces? That is, are there integers $n_{i}$, a sequence $\Sigma_{i}$ of hyperbolic surfaces and $\phi_{i}: \Sigma_{i} \rightarrow \Sigma_{i}$ Teichmüller representatives for the isotopy class $\left[\phi_{i}\right]$ such that the composition

$$
\widetilde{\phi_{i}^{-1}} Z^{n_{i}}: \mathbb{H}^{2} \rightarrow \mathbb{H}^{2}
$$

is a $k_{i}$-quasi-isometry, where $k_{i} \rightarrow 1$ ?

Remark. (1) Implicitly in this question we are choosing an identification of $\mathbb{H}^{2}$ with $\lambda$ and $Z^{n}(\lambda)$. We could do this by choosing some $\alpha \in \pi_{1}(M)$ for which $\alpha Z^{n}(\lambda)$ is very close to $\lambda$ and compose it with a nearest point map, or we could minimize $k_{i}$ over all isometries of $\mathbb{H}^{2}$ to itself. Either method gives the same answer.

(2) One reason to be interested in the existence of such $\phi_{i}$ is that it might help to uniformize $M$. We know that the mapping cylinders of the $\phi_{i}$ are hyperbolic, and therefore estimates as above would give us approximate hyperbolic structures on larger and larger subsets of $\widetilde{M}$. What is lacking is an a priori comparison between the geometry of a singular solv metric on the universal cover of a 3-manifold which fibers over $S^{1}$ and the geometry of the hyperbolic metric, depending only on a bound on the degree of branching on the singular locus, and a bound $c$ on the supremum of the distance from a point in $\widetilde{M}$ to the singular locus. Note that without such a bound, no a priori geometric comparison is possible.

\subsection{Algebraic and analytic geometry of foliations.}

Question 11.4. If $\mathscr{F}$ is a taut foliation, one can let $\gamma_{i}$ be a collection of transverse circles to $\mathscr{F}$ intersecting every leaf and study the space of functions $\mathscr{O}\left(\sum n_{i} \gamma_{i}\right)$ which are leafwise holomorphic, with poles of order at most $n_{i}$ along $\gamma_{i}$. (Here the notation $\sum n_{i} \gamma_{i}$ is meant to suggest a divisor on a Riemann surface). How do these function spaces change as a function of $\gamma_{i}$ ? What is the effect of certain "topological" operations on the $\gamma_{i}$; e.g. crossing changes, cabling etc.

Remark. Ghys used the fact that $\mathscr{O}(D)$ is large for certain $D$ to show that taut foliations admit leafwise embeddings in complex projective space of sufficiently high dimensions. 


\section{COARSE FOLIATIONS}

Question 12.1. Suppose $\rho: \pi_{1}(M) \rightarrow \mathbb{R}$ is a 1-cochain with bounded coboundary; i.e. there is a uniform $C$ so that

$$
|\rho(\alpha)+\rho(\beta)-\rho(\alpha \beta)|<C
$$

for all $\alpha, \beta \in \pi_{1}(M)$. Consider $\pi_{1}(M)$ as a metric space by thinking of it as the vertices of some Cayley graph. Let $L_{\rho}=\rho^{-1}(I) \subset \pi_{1}(M)$.

(1) Are the coarse connected components of $L_{\rho}$ coarsely simply connected?

(2) If the answer is "yes", is there a sense in which $L_{\rho}$ and its translates by $\pi_{1}(M)$ can be thought of as coarse minimal planes in $\pi_{1}(M)$ ?

Remark. (1) If $L_{\rho}$ is coarsely connected for some $I$, we say $\rho$ is weakly uniform, and if it is both coarsely connected and coarsely simply connected, it is uniform. One should think of $L_{\rho}$ as a coarse kernel for $\rho$; under this analogy, coarsely connected corresponds to finitely generated, and coarsely simply connected corresponds to finitely presented.

As one might guess from the Scott core theorem, for $\pi_{1}(M)$ a 3-manifold group, $\rho$ is weakly uniform iff it is uniform ([21]). This may be thought of as a kind of "coarse Stallings fibration theorem", or as a coarsening of Novikov's theorem. In [21] it is also shown that such $\pi_{1}(M)$ either contain $\mathbb{Z} \oplus \mathbb{Z}$, or are word hyperbolic.

(2) The potential usefulness of this question comes from the fact that 1-cochains with bounded coboundary are very common on hyperbolic 3-manifolds. In fact, if the geometrization conjecture is true, every 3 -manifold with infinite $\pi_{1}$ admits infinitely many independent such $\rho$. This is in stark contrast to the fact that many 3 -manifolds with infinite $\pi_{1}$ - even hyperbolic 3 -manifolds - do not admit taut foliations, by [103].

Question 12.2. Does every hyperbolic 3-manifold admit a taut cone field? That is, a cone field $C$ which is recurrent and supports only homotopically essential loops.

Remark. (1) Taut cone fields are introduced in [19]. They exist on small Seifert fibered spaces with infinite $\pi_{1}$ which do not admit essential laminations. Examples of taut cone fields are cone fields supported by pseudo-Anosov flows. However, not every hyperbolic 3-manifold admits a pseudo-Anosov flow. For instance, the Weeks manifold does not admit such a flow ([22]).

(2) Do cone fields stay taut after most surgeries (i.e. those outside finitely many slopes) on a supported loop? Is there a class of examples where the cone field stays taut for all nontrivial surgeries? See also question 9.4.

(3) By Sullivan [109], there is a closed 2-form positive on the de Rham currents supported by a taut cone field. So, for instance, if $M$ is a rational homology sphere, any complete surface (possibly immersed and noncompact) transverse to the cone field enjoys a uniform linear isoperimetric inequality.

\subsection{Coarse invariants; deformation.}

Question 12.3. What deformations of a foliation or lamination should be thought of as "inessential"? For instance - monotone equivalence, cut-and-shear along a surface or transverse lamination, isotopy of branch locus in a branched cover, isomorphic universal circles etc. 
Remark. This question is prompted by the phenomenon that certain geometric objects associated to foliations - e.g. transverse pseudo-Anosov flows - are invariant under quite drastic deformations of the foliation.

An added bonus of a "good" deformation-equivalence class for foliations might be a finite-dimensional parameter space, perhaps analogous to the unit ball of the Thurston norm for finite-depth foliations. It seems that an advantage of the theory of tight contact structures over the theory of taut foliations is a better and more robust equivalence relation. E.g. finiteness up to isotopy of tight contact structures on an atoroidal 3-manifold [56].

\section{NUMERICAL INVARIANTS}

13.1. Godbillon-Vey. The Godbillon-Vey invariant was introduced in [57]. It is a invariance of the foliated cobordism class of a sufficiently smooth $\left(C^{2}\right.$ is sufficient $)$ codimension one foliation of a 3 -manifold. A basic reference for this invariant is [121].

Question 13.1. Suppose $\mathscr{F}$ is a minimal taut $C^{2}$ foliation of an atoroidal 3 -manifold $M$ with

$$
\mathfrak{g v}(\mathscr{F})[M] \neq 0
$$

Is there a choice of 1 -form $\alpha$ with $T \mathscr{F}=\operatorname{ker}(\alpha)$ for which the Godbillon-Vey form $\omega$ (where $d \alpha=\alpha \wedge \omega$ ) has the same sign? i.e. either $\omega \wedge d \omega \geq 0$ everywhere or $\leq 0$ everywhere. Say that such a foliation has monotone wobble.

Remark. (1) Some condition on tori is essential. If $T$ is a torus leaf separating $M$ into two components $M_{1} \amalg M_{2}$, then $\int_{M_{i}} \omega \wedge d \omega$ is independent of the choice of $\alpha$, for each $i$. So we could splice together two foliated manifolds with torus boundary, one with positive Godbillon-Vey invariant, the other with negative invariant in such a way that the sum was nonzero. More generally, if $M_{1}, M_{2}$ are two manifolds foliated by $\mathscr{F}_{1}, \mathscr{F}_{2}$, and $\gamma_{1}, \gamma_{2}$ are transverse circles, the effect of torus connect sum of $M_{1}, M_{2}$ along the $\gamma_{i}$ gives a foliated manifold whose GodbillonVey invariant is the sum of the invariants for $M_{1}$ and $M_{2}$. A form $\alpha$ for which $\omega$ is contact must have $\|\omega \wedge d \omega\|$ potentially very large in a neighborhood of the separating torus $\partial N\left(\gamma_{1}\right)=\partial N\left(\gamma_{2}\right)$.

(2) Minimality is also essential, or else regions of positive and negative twisting can be separated by some exceptional minimal set or even closed leaf. On the other hand, for a minimal foliation, positive twisting can be propagated along neighborhoods of a leafwise path to regions of negative twisting, by analogy with the process of deforming a transitive confoliation to a contact structure.

(3) If $\omega \wedge d \omega \geq 0$ can be achieved everywhere, when can it be achieved so that the zero locus $\omega \wedge d \omega=0$ is a collection of knots, and $M, \omega$ is locally modelled on a branched cover of a contact manifold over a Legendrian link?

(4) If $\omega \wedge d \omega>0$ everywhere - that is, if $\omega$ is actually a contact structure, then in particular $T \mathscr{F}$ admits a nowhere zero section, and the Euler class of $T \mathscr{F}$ is zero. This implies that $\mathscr{F}$ has no closed leaves.

Question 13.2. For $\mathscr{F}$ as in the previous question, suppose there is a choice of $\alpha$ for which $\omega$ is a contact form. Is the contact structure defined by $\omega$ necessarily tight?

13.2. Foliated Gromov norms. For $M, \mathscr{F}$ a foliated manifold, the foliated Gromov norm on $H_{i}$ is the infimum of the $L_{1}$ norm on chains representing homology classes, where we restrict attention to chains which are transverse to $\mathscr{F}$ (in the sense that the induced foliation of each simplex should be affine). A reference for this material is [16]. Notice that to define 
the Godbillon-Vey invariant of $\mathscr{F}$, the transverse structure must be at least Lipschitz + quadratic variation (see [122]); by contrast, the foliated Gromov norm depends only on the homeomorphism type of the foliation.

Question 13.3. Calculate the norm of the fundamental class of a hyperbolic 3-manifold for some taut foliation $\mathscr{F}$ with two-sided branching.

Remark. If $\mathscr{F}$ is $\mathbb{R}$-covered or has one-sided branching, the foliated Gromov norm agrees with the usual Gromov norm.

If some leaf of $\widetilde{\mathscr{F}}$ is asymptotically separated (see question 10.1) then the value of the norm on this fundamental class is strictly greater than the value of the usual Gromov norm on this class.

Question 13.4. Let $\mathscr{F}, \mathscr{G}$ be taut foliations on a hyperbolic manifold $M$. Are there examples where there is a finite cover of $M$ such that a sequence of isotopies of the lift of $\mathscr{G}$ converges geometrically to the lift of $\mathscr{F}$, but no such sequence of isotopies exists in M?

Remark. If this can be done, the foliated Gromov norm of $\mathscr{F}$ is at least as large as that of $\mathscr{G}$ for every homology class.

Question 13.5. Suppose $\mathscr{F}$ is a foliation (possibly $\mathbb{R}$-covered) of a hyperbolic 3-manifold. Define a foliated Gromov norm using cubical chains. Is the value of the foliated norm on the fundamental class always strictly greater than the value of the usual (cubical) Gromov norm?

Remark. Cubical chains cannot necessarily be straightened rel. vertices to be transverse to a given foliation. Suppose $\mathscr{F}$ is a transversely measured foliation. Lift this transverse measure to a function $f: \widetilde{F} \rightarrow \mathbb{R}$. Then for a random regular cube $C$ of side length $t$, the ordering of the vertices of $C$ induced by $f$ converges to a random distribution as $t \rightarrow \infty$.

Question 13.6. What kinds of local order structure are there on a family of deformations of a (taut) foliation? Can one use such structures to define co-ordinates on the "space of deformations" of a taut foliation?

Remark. (1) For instance, local order structure could be provided by numerical invariants, such as the Godbillon-Vey invariant, or foliated Gromov norms.

(2) A family $\mathscr{F}_{t}$ obtained by cut and shear along some particular submanifold, where the re-gluings $f_{t}$ vary monotonically in some sense should be considered a monotone deformation. E.g. in local co-ordinates, the differences $f_{t}-f_{s}$ should be non-negative functions for $t>s$.

\subsection{Invariants for laminations.}

Question 13.7. Is there some notion of a Godbillon-Vey invariant for a lamination?

Remark. One can look at eigendirections of pinching for holonomy and construct a leafwise vector field; a Godbillon-Vey type invariant should be a topological measure of how much this vector field twists in the positive direction. Even an invariant taking values in,,+- 0 would be interesting, where the invariant would be + if for some metric, the transverse twisting ("helical wobble" is Thurston's term) is always positive, - if for some metric it is always negative, and 0 if neither can be achieved. Is this even well-defined? Maybe one would need to restrict attention to the behavior at infinity - e.g. ask for a metric such that for some choice of partition into guts and interstices, the helical wobble over the interstices is always positive. 


\section{IMMERSED OBJECTS}

Question 14.1. Is there a geometric notion for a 3-manifold analogous to LERFness for foliations? What properties could a manifold have so that immersed essential laminations are virtually embedded?

Remark. It might be better to restrict attention to foliations dual to one-cochains with bounded coboundary, since these have a more "algebraic" description.

Question 14.2. Let $\mathscr{F}$ be a taut foliation of $M$. Can leaves of $\mathscr{F}$ be approximated by compact essential surfaces? That is, given a leaf $\lambda$ of $\mathscr{F}$ and a point $p \in \lambda$, is there a sequence of immersed incompressible surfaces $\phi_{i}: \Sigma_{i} \rightarrow M$ and points $p_{i} \in \Sigma_{i}$ such that the images under $\phi_{i}$ of the balls of radius $r_{i}$ about $p_{i}$ where $r_{i} \rightarrow \infty$ converge on compact sets to $p, \lambda$ ?

Remark. (1) If one does not ask for incompressibility, it is easy to find such approximating surfaces; for, if $D \subset \lambda$ is a big embedded subsurface, we can just take two parallel copies of $D$ tubed together by a collection of thin annuli, one for each boundary component of $D$.

(2) If $\pi_{1}(M)$ is LERF, each of the surfaces $\Sigma_{i}$ lifts to an embedded surface in some finite cover. An interesting additional constraint is to ask for these lifts to be Thurston norm minimizing.

(3) Suppose we can choose the $\Sigma_{i}$ which converge uniformly to some leaf $\lambda$ in the sense that for every $\epsilon$ there is an $i$ such that $\left(\phi_{i}\right)_{*} T \Sigma_{i}$ makes an angle of less than $\epsilon$ at every point with $T \lambda$. Then the de Rham cycles $\phi_{i}\left(\left[\Sigma_{i}\right]\right)$ converge to a de Rham cycle supported by $\mathscr{F}$; since $\mathscr{F}$ is taut, this implies $H_{1}(M ; \mathbb{R})$ is nontrivial. On the other hand, the support of the limiting cycle need not be all of $\mathscr{F}$. This can happen, for instance, if the $\Sigma_{i}$ are surfaces of a fibration of $M$ over $S^{1}$ representing homology classes projectively limiting to a class contained in a high codimension face of the Thurston norm. However, if $\lambda$ is dense, every transverse loop to $\mathscr{F}$ is eventually transverse to some $\Sigma_{i}$, and therefore pairs positively with the projective homology class represented by $\Sigma_{i}$. It follows that every transverse loop to $\mathscr{F}$ is nontrivial in homology, and therefore $\mathscr{F}$ is a perturbation of a surface bundle over $S^{1}$.

14.1. Total foliations; families of foliations. A total foliation on an $n$ manifold is a collection of $n$ foliations $\mathscr{F}_{i}$ for $i=1 \ldots n$ which are mutually transverse everywhere and in sufficiently small charts, are topologically conjugate to the total foliation of $\mathbb{R}^{n}$ by translates of the co-ordinate planes.

Question 14.3. What 3-manifolds admit total taut foliations?

Remark. Every 3-manifold admits a total foliation, by a local construction due to Hartdorp [65. Suspensions of Anosov automorphisms of tori admit total taut foliations. Some examples arise in the theory of slitherings ([117]). Anosov flows give rise to some good examples. It is probably better in general for $M$ a hyperbolic manifold to look for taut foliations of $M$ with genuine transverse pseudo-Anosov flows; these exist in very many examples.

Question 14.4. Are there any interesting examples of total genuine laminations?

Remark. (1) One can generalize total foliations to a $\mathscr{G}$-structure where $\mathscr{G}$ is the pseudogroup of homeomorphisms between subsets of $\mathbb{R}^{3}$ which permute translates of 
the co-ordinate planes, but are not required to take horizontal to horizontal, etc. For $M$ a manifold with a $\mathscr{G}$-structure, there is a representation $\pi_{1}(M) \rightarrow \mathrm{S}_{4}$ whose kernel defines a finite cover with a total foliation (in the usual sense). For total laminations, there are local obstructions, much as there are local obstructions to co-orientability. It is reasonable to use this more flexible definition of total lamination, since it encompasses many known examples (e.g. pseudo-Anosov flows transverse to foliations). Is it useful to think of a total genuine lamination as some kind of intrinsically three-dimensional analogue of a pseudo-Anosov flow?

(2) The immersed meridional surface in a cubulation of strictly negative curvature is an example of a total genuine lamination. The complementary regions to a total genuine lamination should be cubes, products of negatively curved polygons with intervals, and polyhedra satisfying the condition of Andre'ev's theorem (see [71]) for all right-angles. It should be straightforward to find a direct proof that manifolds with total genuine laminations admit CAT 0 metrics.

(3) In [81] examples are constructed of hyperbolic manifolds which do not admit nonpositive cubulations. It would be interesting to know whether these manifolds can admit total genuine laminations.

\subsection{Amenability.}

Question 14.5. What is the weakest useful 2-dimensional object that might be present in every atoroidal 3-manifold? For instance, does every hyperbolic 3-manifold $M$ contain an immersed quasigeodesic surface $\Sigma$ of amenable growth?

Remark. (1) Of course, the virtual infinite positive Betti number conjecture would imply that every hyperbolic 3-manifold contains an immersed quasigeodesic compact surface. The point here is to think of as weak a condition as possible, so that there are (hopefully?) many examples to study.

(2) The closure of the image of such an immersed surface $\Sigma$ supports a de Rham cycle by the usual method of Goodman-Plante [58]. If $M$ is a rational homology sphere, then for any loop $\gamma$ in $M$, large subsets of $\Sigma$ must intersect $\gamma$ positively and negatively with approximately equal frequency. Does this imply ergodicity of the geodesic flow?

\section{Miscellaneous}

Question 15.1. What possibilities are there for (co-oriented) laminations in a 3-manifold whose transverse spaces are well-ordered?

Is there a (useful) theory of branched surfaces with ordinal-valued weights?

Remark. Probably the condition that laminations be geometrically realized in a 3-manifold implies that the order types will be countable. There is an evacuation procedure to replace a well-ordered lamination by a simpler one: given $\Lambda$, we can replace $\Lambda$ by $\Lambda_{\infty}$, the sublamination whose leaves are labelled by limit ordinals in a given chart. This will be a proper sublamination. To get an interesting theory, one would want to study laminations for which this procedure does not strictly decrease some notion of complexity - so that the order types should be at least uncountable.

For branched surfaces with no realization issues, more exotic and interesting ordinals could be used; perhaps one can use this to give "geometric" interpretations of interesting theorems in logic. 
Question 15.2. Is there a good notion of taut foliated cobordism? Are there numerical invariants of the equivalence classes this induces on taut foliations which are finer than the Godbillon-Vey invariant?

\section{REFERENCES}

[1] C. Adams, Isometric cusps in hyperbolic 3-manifolds, Mich. Math. J. 463 (1999), 515-531

[2] I. Agol, Lower bounds on volumes of hyperbolic Haken 3-manifolds, preprint, math.GT/9906182

[3] I. Agol and T. Li, An algorithm to detect laminar 3-manifolds, preprint, math.GT/0201310

[4] I. Agol and W. Thurston, personal communication

[5] I. Agol, J. Hass and W. Thurston, The computational complexity of knot genus and spanning area, preprint, math.GT/0205057

[6] L. Ahlfors and L. Bers, Riemann's mapping theorem for variable metrics, Ann. Math. 72 (1960), 413-429

[7] M-T. Benameur, Triangulations and the stability theorem for foliations, Pacific J. Math. 179 (1997), 221239

[8] S. Boyer, D. Rolfsen and B. Wiest, Orderable 3-manifold groups, preprint

[9] M. Brittenham, Essential laminations in Seifert-fibered spaces, Topology, 321 (1993), 61-85

[10] M. Brittenham, Essential laminations and Haken normal form, Pacific J. Math. 1682 (1995), 217-234

[11] M. Brittenham, R. Naimi and R. Roberts, Graph manifolds and taut foliations, J. Diff. Geom. 453 (1997), 446-470

[12] D. Calegari, $\mathbb{R}$-covered foliations of hyperbolic 3-manifolds, Geom. Top. 3 (1999), 137-153

[13] D. Calegari, Foliations transverse to triangulations of 3-manifolds, Comm. Anal. Geom. 81 (2000), 133158

[14] D. Calegari, Foliations and the geometry of 3-manifolds, Dissertation for a PhD at UC Berkeley, (2000)

[15] D. Calegari, The geometry of $\mathbb{R}$-covered foliations, Geom. Top. 4 (2000), 457-515

[16] D. Calegari, The Gromov norm and foliations, Geom. and Func. Anal 10 (2000), 1423-1447

[17] D. Calegari, Almost continuous extension for taut foliations, Math. Res. Lett. 8 (2001), no. 5-6, 637-640

[18] D. Calegari, Foliations with one-sided branching, Geom. Ded. to appear

[19] D. Calegari, Useful branched surfaces which carry nothing, preprint, math.GT/0010146

[20] D. Calegari, Promoting essential laminations, preprint

[21] D. Calegari, Bounded cochains on 3-manifolds, preprint, math.GT/0111270

[22] D. Calegari and N. Dunfield, Laminations and groups of homeomorphisms of $S^{1}$, preprint, math.GT/0203192

[23] A. Candel, Uniformization of surface laminations, Ann. Sci. École Norm. Sup (4) 26 no.4, (1993), 489-516

[24] A. Candel and L. Conlon, Foliations I, AMS Graduate studies in mathematics 23

[25] J. Cantwell and L. Conlon, Every surface is a leaf, Topology 26 (1987), 265-285

[26] J. Cantwell and L. Conlon, Foliation cones, Geom. Top. Monographs 2 Proceedings of the Kirbyfest, 35-86

[27] J. Cannon and W. Thurston, Group invariant Peano curves, preprint

[28] A. Casson and D. Jungreis, Convergence groups and Seifert-fibered 3-manifolds, Invent. Math. 1183 (1994), 441-456

[29] A. Connes, Non-Commutative geometry, Academic Press (1994)

[30] C. Delman and R. Roberts, Alternating knots satisfy strong property P, Comm. Math. Helv. 743 (1999), 376-397

[31] A. Denjoy, Sur les courbes définies par les équations différentiables à la surface du tore, J. Math. Pures Appl. 11 (1932), 333-375

[32] Y. Eliashberg and W. Thurston, Confoliations, AMS

[33] S. Fenley, Depth 1 foliations and end-periodic automorphisms, $\mathrm{PhD}$ thesis at Princeton University, (1992)

[34] S. Fenley, Asymptotic properties of depth one foliations in hyperbolic 3-manifolds, J. Diff. Geom. 362 (1992), 269-313

[35] S. Fenley, Limit sets of foliations in hyperbolic 3-manifolds, Topology 37 no.4 (1998), 875-894

[36] S. Fenley, Foliations with good geometry, Jour. AMS 123 (1999), 619-676

[37] S. Fenley and L. Mosher, Quasigeodesic flows in hyperbolic 3-manifolds, Topology, 40 (2001), no. 3, 530537

[38] S. Fenley, Laminar free hyperbolic 3-manifolds, preprint.

[39] D. Fried, Flow equivalence, hyperbolic systems and a new zeta function for flows, Comment. Math. Helv. 57 no. 2 (1982), 237-259

[40] L. Funar and S. Gadgil, Topological geodesics and virtual rigidity, Alg. Geom. Top. 1 (2001) 369-380

[41] D. Gabai, Foliations and the topology of 3-manifolds, J. Diff. Geom. 18 no. 3, (1983), 445-503 
[42] D. Gabai, Foliations and 3-manifolds, Proceedings of the ICM Vol. 1 (Kyoto, 1990) 609-619

[43] D. Gabai, Convergence groups are Fuchsian groups, J. Diff. Geom. 362 (1992), 269-313

[44] D. Gabai, On the geometrical and topological rigidity of hyperbolic 3-manifolds, Bull. AMS 312 (1994), 228-232

[45] D. Gabai, Problems in foliations and laminations, in Geometric Topology (ed. W. Kazez) proceedings of the 1993 Georgia International Topology Conference; vol. 2 (1997), 1-33

[46] D. Gabai, Essential laminations and Kneser normal form, J. Diff. Geom. 53 no. 3, (1999), 517-574

[47] D. Gabai, Combinatorial volume-preserving flows and taut foliations, Comm. Math. Helv. 751 (2000), 109-124

[48] D. Gabai and W. Kazez, Homotopy, isotopy and genuine laminations of 3-manifolds, in Geometric Topology (ed. W. Kazez) proceedings of the 1993 Georgia International Topology Conference; vol. 1 (1997), 65-77

[49] D. Gabai and W. Kazez, Order trees and laminations of the plane, Math. Res. Lett. 4 (1997), no. 4, 603-616

[50] D. Gabai and W. Kazez, Group negative curvature for 3-manifolds with genuine laminations, Geom. Top. 2 (1998), 65-77

[51] D. Gabai and W. Kazez, The finiteness of the mapping class group for atoroidal 3-manifolds with genuine laminations, J. Diff. Geom. 50 (1998), no. 1, 123-127

[52] D. Gabai and U. Oertel, Essential laminations in 3-manifolds, Ann. Math. (2) 130 no. 2, (1989), 41-73

[53] L. Garnett, Foliations, the ergodic theorem and Brownian motion, J. Func. Anal. 51 (1983), 285-311

[54] E. Ghys, Flots d'Anosov sur les 3-variétés fibrées en cercles, Erg. Theory Dynam. Systems 4 no. 1, (1984), $67-80$

[55] E. Ghys, Topologie des feuilles génériques, Ann. Math. 141 (1995), 387-422

[56] E. Giroux and K. Honda, Finiteness of tight contact structures on 3-manifolds, research announcement

[57] C. Godbillon and J. Vey, Un invariant des feuilletages de codimension 1, Acad. des Sciences. Paris Comptes Rendus. ser. A. 273 (1971), 92-95

[58] S. Goodman and J. Plante, Holonomy and averaging in foliated sets, J. Diff. Geom. 143 (1979), 401-407

[59] P. Griffiths and J. Harris, Principles of algebraic geometry, John Wiley and sons (1978)

[60] M. Gromov, Volume and bounded cohomology, IHES Publ. Math. 56 (1982), 5-99

[61] M. Gromov, Partial differential relations, Springer-Verlag (1986)

[62] A. Haefliger, Structures feuilletées et cohomologie à valeur dans un faisceau de groupoides, Comm. Math. Helv. 32 (1957/58), 248-329

[63] A. Haefliger, Variétés feuilletées, Ann. Scuola Norm. Sup. Pisa 16 (1962), 367-397

[64] W. Haken, Theorie der Normalfächen, Acta Math. 105 (1961), 245-375

[65] D. Hardorp, All compact orientable three dimensional manifolds admit total foliations, Mem. AMS 26 (1980)

[66] K. Hartshorn, Heegaard splittings: The distance complex and the stabilization conjecture, Ph.D. thesis, UC Berkeley (1999)

[67] J. Hass, Minimal surfaces in foliated manifolds, Comm. Math. Helv. 61 (1986), 1-32

[68] A. Hatcher, Measured lamination spaces for 3-manifolds, preprint.

[69] G. Hemion, On the classification of homeomorphisms of 2-manifolds and the classification of 3-manifolds, Acta Math. 142 1-2 (1979), 123-155

[70] J. Hempel, 3-manifolds, No. 86 Ann. Math. Stud. Princeton University Press (1976)

[71] C. Hodgson and I. Rivin, A characterization of compact convex polyhedra in hyperbolic 3-space, Invent. Math. 1111 (1993), 77-111

[72] W. Jaco and H. Rubinstein, 0-Efficient triangulations of 3-manifolds, preprint

[73] M. Jankins and W. Neumann, Rotation numbers of products of circle homeomorphisms, Math. Ann. 2713 (1985), 381-400

[74] T. Kobayashi, Example of hyperbolic knot which do not admit depth 1 foliation, Kobe. J. Math. 132 (1996), 209-221

[75] P. Kronheimer and T. Mrowka, Scalar curvature and the Thurston norm, Math. Res. Lett. 46 (1997), 931937

[76] M. Lackenby, Exceptional surgery curves in triangulated 3-manifolds, preprint; math.GT/9907093

[77] M. Lackenby, Taut ideal triangulations of 3-manifolds, Geom. Top. 4 (2000), 369-395

[78] O. Lehto, Univalent functions and Teichmüller space, GTM 109 Springer-Verlag (1987)

[79] T. Li, Immersed surfaces, Dehn surgery and essential laminations, PhD thesis at Caltech (2000)

[80] T. Li, Laminar branched surfaces in 3-manifolds, Geom. Top. 6 (2002), 153-194

[81] T. Li, Boundary curves of surfaces with 4-plane property, preprint

[82] R. Lickorish, A foliation for 3-manifolds, Ann. Math. 822 (1965), 414-420 
[83] S. Matsumoto, Topological entropy and Thurston's norm of atoroidal surface bundles over the circle, J. Fac. Sci. Univ. Tokyo Sect. 1A Math. 34 no. 3, (1987), 763-778

[84] S. Matsumoto, Some remarks on foliated $S^{1}$ bundles, Invent. Math. 90 no. 2, (1987), 343-358

[85] C. McMullen, Renormalization and 3-manifolds which fiber over the circle, No. 142 Ann. Math. Stud. Princeton University Press (1996)

[86] C. McMullen, Polynomial invariants for fibered 3-manifolds, Ann. Scient. École Norm. Sup. 33 (2000), $519-560$

[87] G. Meigniez, Bouts d'un groupe opérant sur la droite: 2. applications á la topologie des feuilletages, Tóhoku Math. J. 43 (1991), 473-500

[88] J. Milnor, On the existence of a connection with curvature zero, Comm. Math. Helv. 32 (1958), 215-223

[89] E. Moise, Geometric topology in dimensions 2 and 3, Springer-Verlag GTM 47 (1977)

[90] L. Mosher, Surfaces and branched surfaces transverse to pseudo-Anosov flows on 3-manifolds, Jour. Diff. Geom. 34 (1991), 1-36

[91] L. Mosher, Dynamical systems and the homology norm of a 3-manifold, I: Efficient intersection of surfaces and flows, Duke Math. Jour. 65 no. 3 (1992), 449-500

[92] L. Mosher, Dynamical systems and the homology norm of a 3-manifold, II, Invent. Math. 107 (1992), 243281

[93] L. Mosher, Laminations and flows transverse to finite depth foliations. Part $i$ : branched surfaces and dynamics, preprint

[94] R. Naimi, Foliations transverse to fibers of Seifert manifolds, Comment. Math. Helv. 69 no. 1 (1994), 155 162

[95] S. Novikov, Topology of foliations, Trud. Mosc. Math. Ob. 14 (1965), 268-304

[96] U. Oertel, Homology branched surfaces: Thurston's norm on $\mathrm{H}_{2}\left(M^{3}\right)$, Low dimensional topology any Kleinian groups; LMS No. 112 (1986), 253-272

[97] C. Palmeira, Open manifolds foliated by planes, Ann. Math. 107 (1978), 109-131

[98] H. Pittie, Characteristic classes of foliations, Research Notes in Mathematics 10, Pitman Publishing (1976)

[99] J. Pitts, Existence and regularity of minimal surfaces on Riemannian manifolds, Princeton University Press Mathematical Notes 27 (1981)

[100] D. Pixton, Nonsmoothable, nonstable group actions, Trans. AMS 229 (1977), 259-268

[101] H. Poincaré, Sur les courbes définies par les équations différentielles, J. de Mathématiques 14, (1885)

[102] G. Reeb, Sur certaines proprietes topologiques des varietes feuilletees, Act. Sci. Indust. 1183 (1952), 91-158

[103] R. Roberts, J. Shareshian and M. Stein, Infinitely many hyperbolic 3-manifolds which contain no Reebless foliation, preprint.

[104] D. Rolfsen, Knots and Links, Publish or Perish (1976)

[105] H. Rosenberg, Foliations by planes, Topology 7 (1968), 131-138

[106] H. Rubinstein, Minimal surfaces in geometric 3-manifolds, preprint

[107] S. Shields, Branched surfaces and the simplest foliations of 3-manifolds, Pac. J. Math. 1772 (1997), 305-327

[108] D. Stroock, Probability theory - an analytic view, Cambridge Univ. Press (1993)

[109] D. Sullivan, Cycles for the dynamical study of foliated manifolds and complex manifolds, Invent. Math. 36 (1976), 225-255

[110] D. Sullivan, A homological characterization of foliations consisting of minimal surfaces, Comm. Math. Helv. 54 (1979), 218-223

[111] W. Thurston, Foliations of 3-manifolds that are circle bundles, PhD thesis at UC Berkeley (1972)

[112] W. Thurston, Noncobordant foliations of $S^{3}$, Bulletin of the AMS 78 no. 4, (1972), 511-514

[113] W. Thurston, A local construction of foliations for 3-manifolds, Proc. Symp. Pure Math. XXVII (part I) (1973), 315-319

[114] W. Thurston, The geometry and topology of three-manifolds, Lecture notes from Princeton University 1978-1980

[115] W. Thurston, A norm for the homology of 3-manifolds, Mem. AMS 59 (1986) no. 339, pp. i-vi and 99-130

[116] W. Thurston, On the geometry and dynamics of diffeomorphisms of surfaces, Bull. AMS. 192 (1988), 417-431

[117] W. Thurston, Three-manifolds, Foliations and Circles, I, eprint math.GT/9712268

[118] W. Thurston, Three-manifolds, Foliations and Circles, II, unfinished manuscript

[119] W. Thurston, personal communication

[120] T. Tsuboi, Homology of diffeomorphism groups, and foliated structures, translated from the Japanese in Sugaku Expositions 3 no. 2, (1990), 145-181 
[121] T. Tsuboi, Characterization of Godbillon-Vey classes, translated from the Japanese in Sugaku Expositions 8 no. 2, (1995), 165-182

[122] T. Tsuboi, Homological and dynamical study on certain groups of Lipschitz homeomorphisms of the circle, J. Math. Soc. Jap. 471 (1995), 1-30

[123] J. Wood, Foliations on 3-manifolds, Ann. Math. 89 (1969), 336-358

[124] A. Zeghib, Sur les feuilletages géodésiques continus des variétés hyperboliques, Invent. Math. 114 no.1 (1993), 193-206

[125] A. Zeghib, Lipschitz regularity in some geometric problems preprint

Department of Mathematics, California Institute of Technology, Pasadena CA 91125

E-mail address: dannyc@its.caltech.edu 